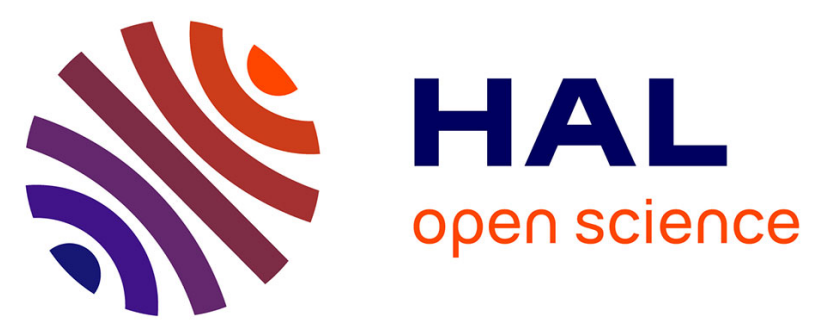

\title{
Derivation of the Young's and shear moduli of single-walled carbon nanotubes through a computational homogenization approach
}

Elie El Khoury, Tanguy Messager, Patrice Cartraud

\section{- To cite this version:}

Elie El Khoury, Tanguy Messager, Patrice Cartraud. Derivation of the Young's and shear moduli of single-walled carbon nanotubes through a computational homogenization approach. International Journal for Multiscale Computational Engineering, 2011, 9 (1), pp.97-118. 10.1615/IntJMultCompEng.v9.i1.80 . hal-01006722

\section{HAL Id: hal-01006722 \\ https://hal.science/hal-01006722}

Submitted on 1 Dec 2016

HAL is a multi-disciplinary open access archive for the deposit and dissemination of scientific research documents, whether they are published or not. The documents may come from teaching and research institutions in France or abroad, or from public or private research centers.
L'archive ouverte pluridisciplinaire HAL, est destinée au dépôt et à la diffusion de documents scientifiques de niveau recherche, publiés ou non, émanant des établissements d'enseignement et de recherche français ou étrangers, des laboratoires publics ou privés.

\section{(c)(1)}

Distributed under a Creative Commons Attribution| 4.0 International License 


\title{
DERIVATION OF THE YOUNG'S AND SHEAR MODULI OF SINGLE-WALLED CARBON NANOTUBES THROUGH A COMPUTATIONAL HOMOGENIZATION APPROACH
}

\author{
Elie El Khoury, ${ }^{1}$ Tanguy Messager, ${ }^{2}$ \& Patrice Cartraud ${ }^{1}$
}

\author{
${ }^{1}$ Institut de Recherche en Génie Civil et Mécanique (UMR CNRS 6183), Ecole Centrale de \\ Nantes, BP 92101, 44321, Nantes Cédex3, France \\ ${ }^{2}$ Laboratoire de Mécanique de Lille (UMR CNRS 8107), Université Lille 1, Cité Scientifique, \\ 59655 Villeneuve d'Ascq Cedex, France
}

In this study, the computation of the traction-torsion-bending behavior of single-walled carbon nanotubes (SWCNTs) is investigated. A structural mechanics model is used to describe the response of the nanotube; the atomic interactions are represented with $3 D$ beams. Nanotubes are slender structures, taking benefit from their axial periodicity or helical symmetry. Homogenization theory is used to obtain their overall beam behavior from the solution of basic cell problems. These problems are solved through a finite element approach and involve concise models, whatever the SWCNT type. The computed results show that the bending behavior appears to be decoupled from the axial one and independent of the moment direction. Young's and shear moduli are derived, and it is shown that the Young's moduli are very close in traction and bending. Comparisons with the data in the literature reveal good agreements. Finally, scale effects are studied, and the moduli of the SWCNTS are compared to those of the graphene, thus demonstrating mechanical sensitivity to curvature.

KEY WORDS: SWCNTs, homogenization, helical symmetry, periodic structures, Young's modulus, shear modulus

\section{INTRODUCTION}

Carbon nanotubes (CNTs) can be described as lengthy nanostructures (with nanometer diameters and micrometer lengths) of connected carbon atoms forming straight tubular atomic bond networks. Since the single-walled carbon nanotubes (SWCNTs) were discovered by Iijima in 1991, they have been the focus of considerable interest in scientific communities. Such great attention is due in particular to their remarkable electrical, thermal, and also mechanical properties, such as strength and stiffness. Therefore, CNTs have been involved in numerous recent research studies dealing with low-weight structural nanoreinforced composite materials, nanorobotics, and nanoengineering developments.

Such CNT applications require a sharp knowledge of their mechanical response. Therefore, the determination of elastic moduli of SWCNTs has been the subject of much research, including experimental techniques and modeling and numerical approaches, e.g., the review papers Qian et al. (2002), Srivastava et al. (2003), and Rafii-Tabar (2004). Of the modeling approaches, the main categories which can be found are ab initio, molecular dynamics, tight-binding molecular dynamics, and continuum mechanics. The latter involves a structural mechanics approach and continuum shell modelling. The results obtained from these different approaches will be exposed and discussed in the present paper. 


\section{NOMENCLATURE}

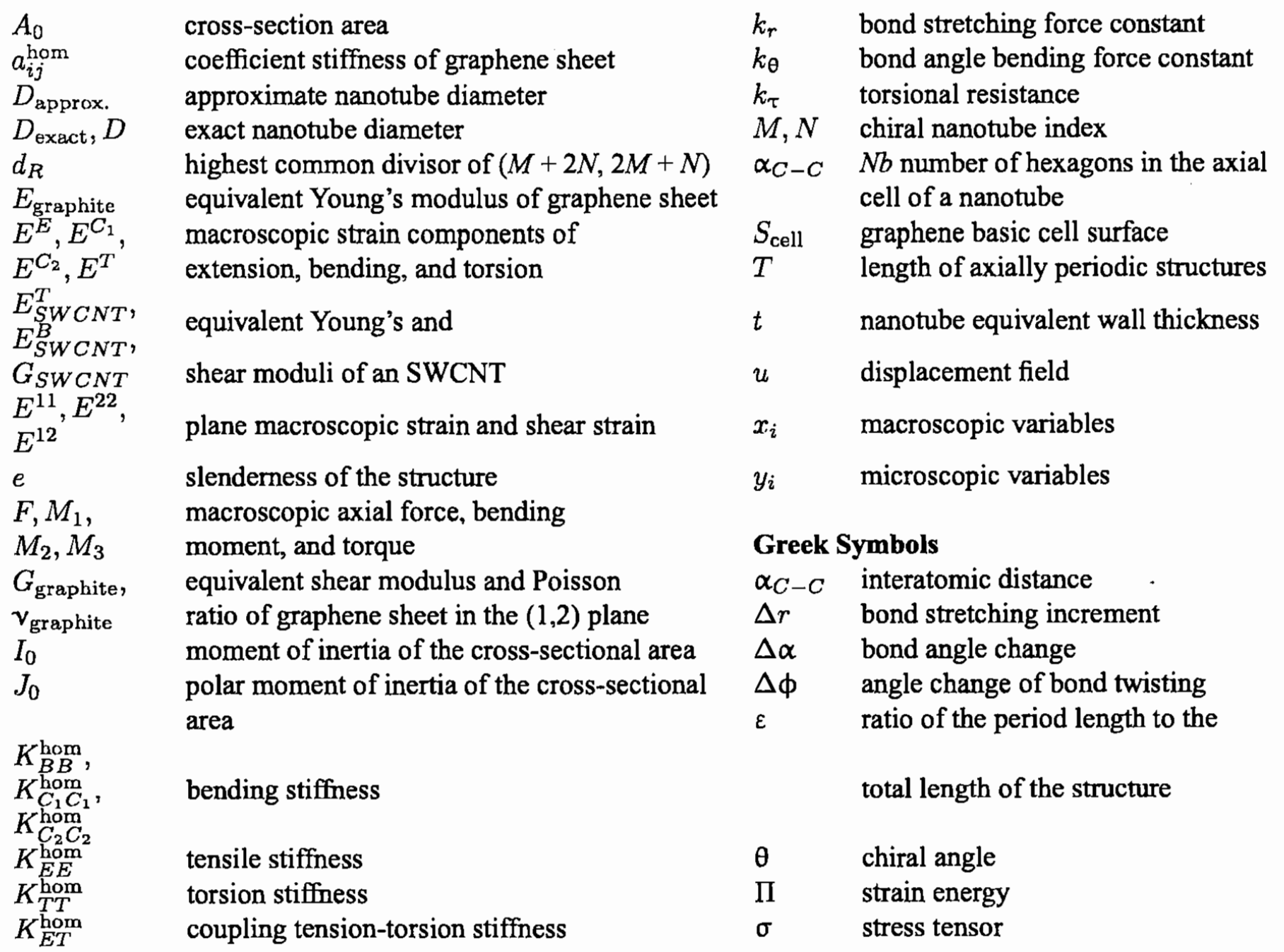

The objective and contribution of the present study is to propose a computational homogenization approach. In the first step, and following a discrete description of such tubular atomic networks, the SWCNTs are modeled as space-frame structures, using straight 3D beams representing the carbon atomic covalent links. The choice of this modeling approach-instead of an atomistic model-is justified because of the use of a homogenization theory which was initially developed in a structural mechanics framework. Moreover, the coupling of this homogenization theory and a solution approach such as the finite element method is very easy, and in the literature, numerical applications appear to be limited, especially in the case of nanotubes. By applying this homogenization procedure based on the asymptotic expansion method, the overall mechanical beamlike behavior is derived from the solution of basic cell problems, taking benefit from the repetitive hexagonal atomic bonds patterns. These problems are solved through a finite element (FE) approach, which involves very concise axial or helical basic cell FE models for the computation of the tension-torsion-bending, beamlike behavior of SWCNTs. This approach was performed in a previous work by Messager and Cartraud (2008), where the study was restricted to the axial behavior of armchair and zigzag SWCNTs. In this paper, the latter approach is applied to SWCNTs of arbitrary chirality. Moreover, in addition to the overall axial behavior (traction and torsion), the bending behavior is also investigated. Once the overall beam behavior is known, 
an axial Young modulus can be derived from its axial or bending behavior, and a shear modulus from the torsional stiffness. The traction and bending Young's moduli are compared. Finally, the results are processed by performing comparisons with the stiffness of the graphene sheet in order to evaluate the scale effects related to diameter size and their evolution with the chirality.

In Section 2 this paper presents the geometrical description of SWCNTs, detailing the covalent link network and diameter calculation. In Section 3 the overall mechanical elastic response of the SWCNT is introduced, relating the macroscopic strain components and loadings. Next, a brief bibliography survey details the approaches devoted to the determination of the SWCNT mechanical properties, as well as results obtained from analytical, computational, and experimental techniques. Next, Section 4 describes the developed homogenization procedure and its corresponding numerical FE implementation. The results obtained from this approach, for the SWCNT elastic behavior, are presented in Section 5. They are expressed in terms of beam stiffnesses (traction-torsion-bending) or material moduli and for several chirality types, and are compared with literature data. The study of the scale effects is discussed next. Finally, concluding remarks are given in Section 6.

\section{NANOTUBE DESCRIPTION}

The single-walled carbon nanotubes (SWCNTs) are rolled-up tubular shells of graphene sheet. The latter is a plane sheet of carbon atoms where interatomic links form a hexagonal pattern as schematized in Fig. 1; this hexagonal pattern is repeated periodically, leading to the binding of each carbon atom to three neighboring atoms with covalent bonds. These covalent bonds are strong chemical links and play a major role in the impressive mechanical properties of graphite, and of all carbon-related nanostructures.

The single-walled carbon nanotubes (SWCNTs) can be viewed as a graphene sheet that has been rolled into a cylinder of radius $R$ by joining the two points $O$ and $O^{\prime}$, represented in Fig. 1. The atomic structure of a nanotube depends on the chirality, which can be represented by the chiral vector $\overrightarrow{O O}^{\prime}$, expressed as a linear combination of the unit translational vectors in the hexagonal pattern

$$
\overrightarrow{O O}^{\prime}=M \cdot \vec{a}_{M}+N \cdot \vec{a}_{N}
$$

where $\vec{a}_{M}$ and $\vec{a}_{N}$ are the basic vectors of the hexagonal pattern, and the positive integers $M$ and $N$ are the chiral index numbers. The corresponding chiral angle $\theta$ depicted in Fig. 1 is the angle between the chiral vector $\overrightarrow{O O}^{\prime}$ and the basic vector $\left(\vec{a}_{M}+\vec{a}_{N}\right)$ and is defined as follows:

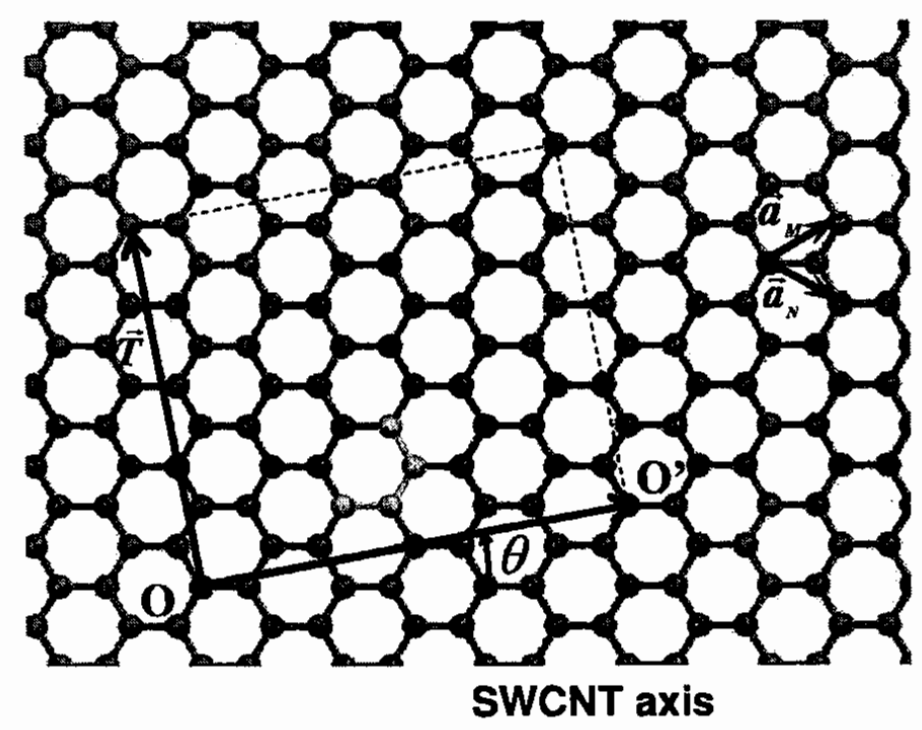

FIG. 1: Plane sheet of graphene with SWCNT $(4,2)$ chiral parameters 


$$
\theta=\arctan \left(\frac{M-N}{(M+N) \sqrt{3}}\right) .
$$

The SWCNTs can be classified as follows:

(1) armchair, when $N=M$, leading to $\theta=0^{\circ}$;

(2) zigzag, with $N=0$, inducing $\theta=30^{\circ}$;

(3) chiral, where $N \neq 0$ and $N \neq M$, thus leading to $0^{\circ}<\theta<30^{\circ}$ when $M>N$, and $-30^{\circ}<\theta<0$ when $M<N$.

As discussed in Sections 3.1 and 3.2, an SWCNT exhibits a beamlike behavior. Therefore, since the objective is to derive material parameters such as Young's and shear moduli from this beamlike behavior, the definition of the geometrical properties of the SWCNT's cross section should be studied carefully. The interatomic links between the carbon atoms represented in Fig. 1 remain straight in the rolled-up tubular geometry of an SWCNT. Consequently, the cross section of a nanotube is polygonal, which is obvious for cross sections with small diameters, as shown in Fig. 2. For a polygonal section (see Fig. 2), it is possible to define an exact diameter $D_{\text {exact }}$ as the ratio of its circumference to the number $\pi$. The value of this diameter is found by solving the following nonlinear equation:

$$
(M+N) \arcsin \left(\frac{\alpha_{C-C} \cos \theta}{D_{\text {exact }}}\right)+M \arcsin \left(\frac{\alpha_{C-C} \cos \left(\frac{\pi}{3}-\theta\right)}{D_{\text {exact }}}\right)+N \arcsin \left(\frac{\alpha_{C-C} \cos \left(\frac{\pi}{3}+\theta\right)}{D_{\text {exact }}}\right)=\pi
$$

However, an approximation for SWCNT diameters can be found in the literature [e.g., Tserpes and Papanikos (2005), Barros et al. (2006)], which is assumed to be circular by using the relation

$$
D_{\text {approx. }}=\frac{O O^{\prime}}{\pi}=\frac{\alpha_{C-C}}{\pi} \sqrt{3\left(M^{2}+N^{2}+M N\right)} .
$$

Figure 3 illustrates the variation of the SWCNT diameters for the three series of chiral nanotubes with respect to the chiral index $M$. Moreover, it illustrates the comparison between the exact diameter $D_{\text {exact }}$ and its approximation $D_{\text {approx. }}$. It can be observed that the polygonal section for a large diameter is similar to the circular section.

Finally, the multiwalled carbon nanotubes (MWCNTs) are composed of sets of coaxially situated SWCNTs of growing radius (Dresselhaus et al., 2001). CNTs can be depicted as lengthy cylinders with nanometer diameters and micrometer lengths. In the present study we are interested by their overall mechanical behavior, without taking into account the end effects.
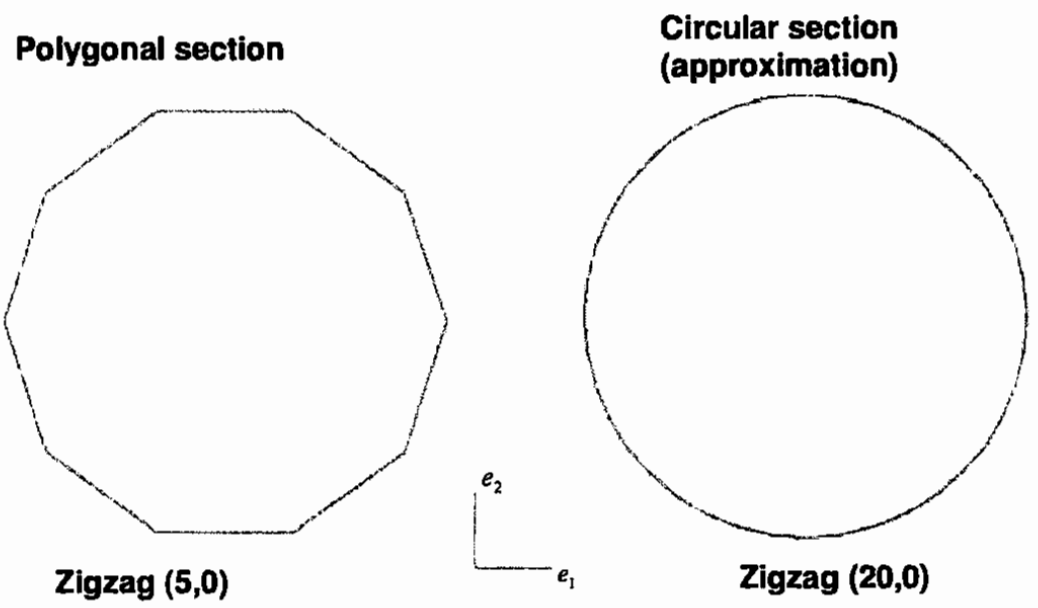

FIG. 2: Cross-sectional of $(5,0)$ and $(20,0)$ CNTs 


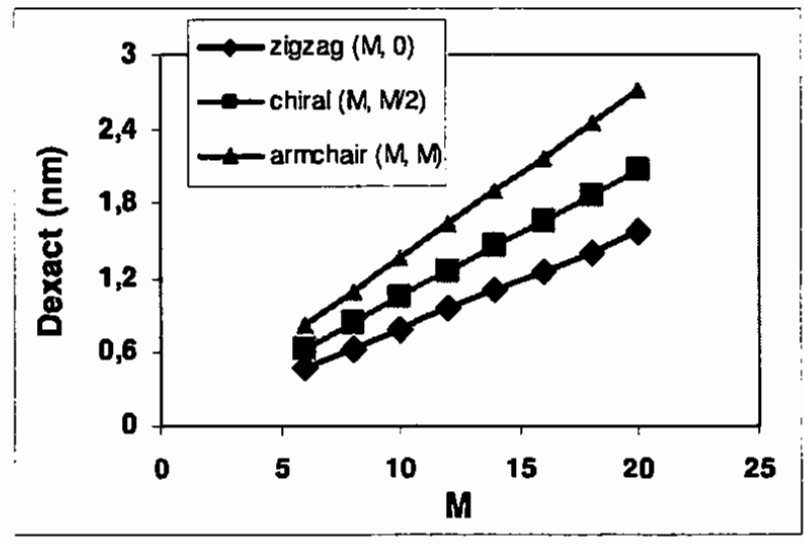

(a)

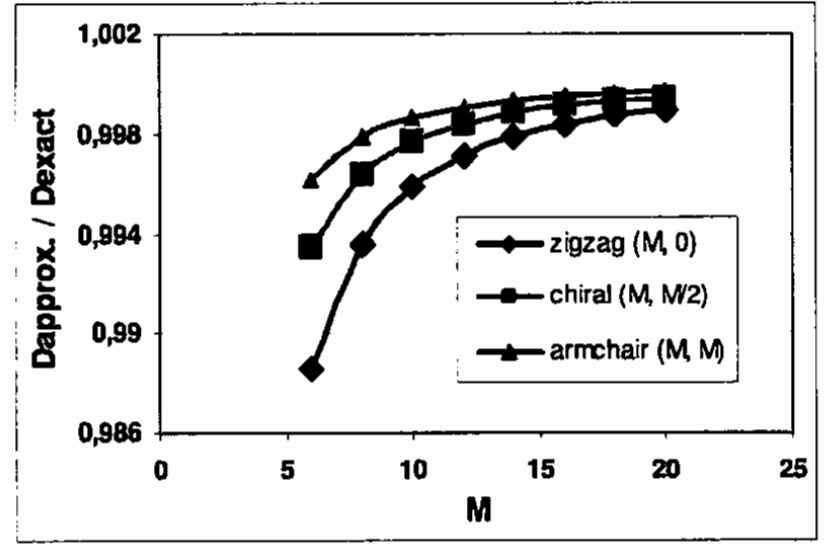

(b)

FIG. 3: Variation of the CNTs diameter with the chiral index $M$

\section{OVERALL MECHANICAL PROPERTIES}

\subsection{SWCNT Beam Properties}

Several approaches have been discussed in the literature to compute the overall elastic mechanical properties of SWCNTs. In most of them, the properties are derived from the study of a structure consisting of a large part of the nanotube (Li and Chou, 2003).

Since the nanotube is a slender structure, it is expected to behave as a beamlike structure. This result can be justified by the asymptotic expansion method, which is a classical approach in the framework of continuum mechanics [see Trabucho and Viaño (1996), which is a major reference in this field]. This method has been used for various applications in the literature [see Dallot et al. (2009) for limit analysis of periodic beams, or Boutin and Hans (2003, 2008) and Moustaghfir et al. (2007) for studying their dynamics]. Another method is proposed in Geers et al. (2007), in which the overall beam behavior is assumed, and then the localization problem is formulated on the representative volume element.

In this work the asymptotic expansion method is used, and it will be proved in Section 4.2 that the macroscopic behavior corresponds to an anisotropic Navier-Bernoulli beam theory, as shown in Fig. 4. Hence, the macroscopic beam behavior (including traction, bending, and torsion) of the nanotube can be expressed under the following form:

$$
\left\{\begin{array}{l}
F \\
M_{1} \\
M_{2} \\
M_{3}
\end{array}\right\}=\left[K^{\text {hom }}\right]\{E\}=\left[\begin{array}{cccc}
K_{E E}^{\text {hom }} & K_{E C_{1}}^{\text {hom }} & K_{E C_{2}}^{\text {hom }} & K_{E T}^{\text {hom }} \\
K_{C_{1} E}^{\text {hom }} & K_{C_{1} C_{1}}^{\text {hom }} & K_{C_{1} C_{2}}^{\text {hom }} & K_{C_{1} T}^{\text {hom }} \\
K_{C_{2} E}^{\text {hom }} & K_{C_{2} C_{1}}^{\text {hom }} & K_{C_{2} C_{2}}^{\text {hom }} & K_{C_{2} T}^{\text {hom }} \\
K_{T E}^{\text {hom }} & K_{T C_{1}}^{\text {hom }} & K_{T C_{2}}^{\text {hom }} & K_{T T}^{\text {hom }}
\end{array}\right]\left\{\begin{array}{c}
E^{E} \\
E^{C_{1}} \\
E^{C_{2}} \\
E^{T}
\end{array}\right\},
$$

where $\left[K^{\text {hom }}\right]$ is symmetric and the $K_{i j}^{\text {hom }}\left(i, j=E, C_{1}, C_{2}, T\right)$ matrix terms are the homogenized stiffness coefficients, relating the axial force $F$, the bending moments $M_{1}, M_{2}$, and the torque $M_{\mathrm{z}}$ (represented in Fig. 4) to the overall strain components of extension, curvature, and torsion noticed in $E^{E}, E^{C_{1}}, E^{C_{2}}$, and $E^{T}$ (Cartraud and Messager, 2006).

However, most of the time in the literature, the SWCNT elastic properties are given under the form of the Young and shear moduli. Therefore, in order to derive from the beam the stiffness matrix of Eq. (3.1) equivalent elastic moduli, one has to consider the SWCNT as a solid cylinder. The geometrical properties of this cylinder of diameter $D_{\text {exact }}$ [see Eq. (2.3)] are denoted $A_{0}$ for its cross-sectional area, $I_{0}$ for its moment of inertia, and $J_{0}$ for its polar moment of inertia. Then, one can define the equivalent traction Young's modulus $E_{S W C N T}^{T}$ by

$$
E_{S W C N T}^{T}=\frac{K_{E E}^{\text {hom }}}{A_{0}} .
$$




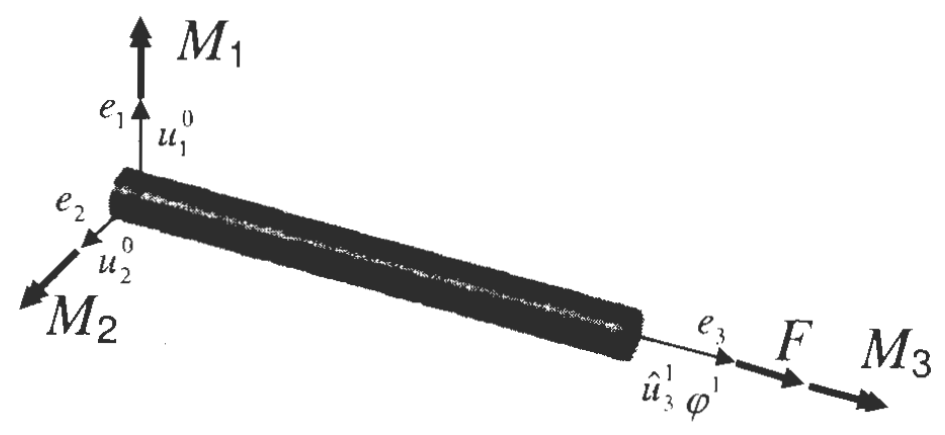

FIG. 4: Loading and displacements of a $e_{3}$-axis beamlike structure

Since we will prove later that $K_{C_{1} C_{1}}^{\text {hom }}=K_{C_{2} C_{2}}^{\text {hom }}$, the bending Young's modulus is defined by

$$
E_{S W C N T}^{B}=\frac{K_{C_{1} C_{1}}^{\mathrm{hom}}}{I_{0}} .
$$

Similarly, the shear modulus of SWCNTs is obtained using the following relation:

$$
G_{S W C N T}=\frac{K_{T T}^{\mathrm{hom}}}{J_{0}} .
$$

The problem is now to define the geometrical characteristics $A_{U}, I_{0}$, and $J_{0}$, and it raises the question of the definition of thickness of the cylinder. We share the point of view of several authors (Huang et al., 2006 and Tu and Ou-Yang, 2008), according to which we cannot define the wall thickness in a unique way. Huang et al. (2006) have investigated the effective wall thickness of the graphene sheet and SWCNTs from interatomic potential. They found that SWCNTs have a thickness ranging from 0.06 to $0.09 \mathrm{~nm}$, and it depends on the type of loading. Tu and Ou-Yang (2008) listed three methods to find the wall thickness of SWCNTs. The first one is around $0.07 \mathrm{~nm}$, derived from continuum shell modeling. The second one is about $0.14 \mathrm{~nm}$, calculated from molecular dynamics. The third one is equal to $0.34 \mathrm{~nm}$, which is the interlayer spacing of the graphene sheet. In the following, we use this latter value, as did Dresselhaus et al. (2001) and Li and Chou (2003).

\subsection{A Brief Literature Survey}

Many experimental and numerical studies were performed to obtain the elastic moduli of SWCNTs and the graphene sheet.

Numerous experimental investigations on the mechanical properties of carbon nanotubes can be cited. Krishnan et al. (1998) found that the average value of Young's modulus is $1.3 \mathrm{TPa}$ for 27 SWCNTs by measuring thermal vibrations using transmission electron microscopy. ln order to determine the elastic properties from deflection versus force measurements, a technique of atomic force microscopic was used by Salvetat et al. (1999); they reported that the Young's modulus of SWCNT is $1 \mathrm{TPa}$. Due to the difficulty in experimental techniques arising from the very small size of SWCNTs, to the best of our knowledge, there is no report on the measured values of the shear modulus of carbon nanotubes.

Some research works are based on numerical atomistic modeling approaches to investigate the mechanical properties of carbon nanotubes. The atomistic approaches include the ab initio method, density functional theory (DFT), atomistic molecular dynamics (MD), and tight-binding molecular dynamics (TB).

The $\mathrm{ab}$ initio is a simulation method to directly solve the complex quantum many-body Schrodinger equation using numerical algorithms. Current ab initio simulation methods are based on a rigorous mathematical foundation of the DFT. The Young's modulus and Poisson's ratio have been computed using an ab initio method by Van Lier et al. (2000); the corresponding values found are $1.14 \mathrm{TPa}$ and 0.11 , respectively. The MD refers most commonly to the situation where the motion of atoms or molecules is treated in approximate finite difference equations of Newtonian 
mechanics (Wenxing et al., 2004; Agrawal et al., 2006). Atomistic studies of SWCNTs can find the surface Young's modulus of the nanotube, which is the product of Young's modulus and nanotube thickness $t$. The TB is based on the combined use of features in ab initio and MD. For example, Zhang and Dumitrica (2008) obtained an effective wall thickness equal to $0.08 \mathrm{~nm}$ and a Young's modulus of around 5.2 TPa. $\mathrm{Lu}$ (1997) calculated the Young's modulus of SWCNTs and the graphene sheet, subjected to axial strain, to be around 0.974 and $1.02 \mathrm{TPa}$, respectively. The elastic moduli for SWCNTs are shown to be insensitive to nanotube diameter and chirality because an empirical pair potential was used. However, Hernandez et al. (1998), by employing a nonorthogonal TB theory, showed the evolution of the Young's modulus with nanotube diameter and chirality and gave an average value of $1.24 \mathrm{TPa}$. It was found that the Young's modulus is noticeably dependent on the nanotube diameter, as the tube diameter is small. Gupta and Batra (2008) have used molecular mechanics to study the free vibrations of the relaxed configuration of an SWCNT to calculate the elastic properties of SWCNTs. Gupta et al. (2005) calculated the elastic moduli by applying pressure along the tube axis using the Tersoff-Brenner potential.

In the literature, the elastic moduli of carbon nanotubes has also been studied in the framework of continuum mechanics. These approaches replace discrete molecular structure with continuum models. In Odegard et al. (2002), a nanotube carbon is modeled by many truss members. Such approaches appear to be more efficient from a computational point of view. Using the harmonic potential, $\mathrm{Li}$ and Chou (2003) and Tserpes and Papanikos (2005) presented a structural mechanics approach to model the deformation of SWCNTs, described as geometrical space-frame structures. Young's and shear moduli have been calculated by applying tensile force and torsional moment, respectively, on one end and fixing the other end. Using the same approach, To (2006) derived the Poisson ratio from the traction response. Their results show that SWCNTs' mechanical properties depend strongly on tube diameter and weakly on chirality. Assuming a wall thickness equal to that of graphene $(0.34 \mathrm{~nm})$, they found an average value of Young's and shear moduli of 1.04 and $0.48 \mathrm{TPa}$, respectively. Using a similar approach, by incorporating a modified Morse potential, Meo and Rossi (2006) calculated the Young's modulus of the SWCNTs and the graphene sheet by modeling the interactions between the atoms with linear and torsion springs. In order to compute their Young's modulus, all degrees of freedom of the one extremity were restrained while a displacement was imposed in the opposite extremity. On the other hand, Kalamkarov et al. (2006) derived the elastic properties of SWCNTs by modeling the carbon nanotubes as a continuum shell using the asymptotic homogenization method. Their elastic properties were investigated under the action of pure tension and pure torsion. Chang et al. (2005) and Natsuki et al. (2004) used an analytical molecular structural mechanics approach to study the mechanical properties of a carbon nanotube subjected to axial loadings. In their work, analytical expressions for the elastic moduli and Poisson ratio of SWCNTs have been deduced as a function of the nanotube diameter. Using an analytical molecular structural mechanics approach, Xiao et al. (2006) studied the elastic properties of SWCNTs under radial pressure. By combining the methods of molecular mechanics and continuum mechanics, Wu et al. (2006) derived the elastic response of armchair and zigzag SWCNTs and the graphene sheet by considering an SWCNT as a thin cylinder subjected to an axial tension loading or torsion loading. The tube thickness used in their work is equal to $0.258 \mathrm{~nm}$. For a given tube diameter, the Young's modulus for armchair nanotubes is stiffer than for zigzag nanotubes. However, the shear modulus for zigzag nanotubes is slightly larger than that for armchair nanotubes.

The results of the overall elastic mechanical behavior of the SWCNTs and the graphene sheet are summarized in Tables 1 and 2 for the different cited works found in the literature.

In Table 1, note that the values of the Young's modulus depend on the tube wall thickness $t$. The smaller the wall thickness, the higher the Young's modulus calculated. The Young's modulus and the wall thickness $t$ of SWCNTs are not well-defined physical quantities, as mentioned previously in Section 3.1. However, the surface Young's modulus has the same value. Thus it is a better-defined quantity than the Young's modulus and the tube wall thickness as reported in Tu and Ou-Yang (2008) and Huang et al. (2006).

\section{COMPUTATIONAL HOMOGENIZATION METHODOLOGY}

In this paper, the homogenization theory is applied to determine the overall behavior of CNTs. In the first step, the mechanical response of the nanotube is modeled by using a structural mechanics approach with beams linking the neighboring atoms. 
TABLE 1: Comparison of traction Young's modulus, shear modulus, and Poisson ratio results for different values of wall thickness

\begin{tabular}{|c|c|c|c|c|c|}
\hline \multirow{2}{*}{ Authors } & \multirow{2}{*}{ Method } & \multicolumn{4}{|c|}{ Elastic moduli and wall thickness } \\
\hline & & $\begin{array}{l}t \\
\text { (nm) }\end{array}$ & $\begin{array}{l}E_{S W C N T}^{T} \\
\text { (TPa) }\end{array}$ & $\begin{array}{l}G_{S W C N T} \\
\text { (TPa) }\end{array}$ & $v$ \\
\hline \multicolumn{6}{|c|}{ Numerical or theoretical studies } \\
\hline $\mathrm{Lu}(1997)$ & $\mathrm{MD}$ & 0.34 & 0.974 & - & 0.28 \\
\hline Hernandez et al. (1998) & TB & 0.34 & 1.24 & - & - \\
\hline Van Lier et al. (2000) & Ab-initio & 0.34 & 1.14 & - & 0.11 \\
\hline Odegard et al. (2002) & Equivalent continuum modeling & 0.69 & - & - & - \\
\hline $\mathrm{Li}$ and Chou (2003) & Structural mechanics & 0.34 & 1.038 & 0.48 & - \\
\hline Natsuki et al. (2004) & Molecular structural mechanics & 0.34 & $1.1-0.73$ & $0.86-0.55$ & 0.27 \\
\hline Pantano et al. (2004) & Continuum shell modeling & 0.075 & 4.75 & - & - \\
\hline Chang et al. (2005) & Molecular structural mechanics & 0.34 & 1.06 & - & 0.16 \\
\hline Gupta et al. (2005) & Hydrostatic pressure & 0.34 & 1.223 & 0.3281 & - \\
\hline Tserpes and Papanikos (2005) & Structural mechanics & 0.34 & 1.05 & 0.485 & \\
\hline Agrawal et al. (2006) & $\mathrm{MD}$ & 0.34 & $0.73-0.82$ & - & - \\
\hline \multirow[t]{2}{*}{ Kalamkarov et al. (2006) } & Asymptotic homog. model & 1.29 & 1.44 & 0.27 & - \\
\hline & Structural mechanics & 0.68 & $0.97-1.05$ & $0.14-0.47$ & - \\
\hline Meo and Rossi (2006) & Structural mechanics & 0.34 & 0.92 & - & - \\
\hline To (2006) & Structural mechanics & 0.34 & 1.03 & 0.47 & - \\
\hline Xiao et al. (2006) & Molecular structural mechanics & 0.34 & $1-1.2$ & - & 0.16 \\
\hline Wu et al. (2006) & Molecular mechanics & 0.258 & 1.06 & 0.418 & 0.27 \\
\hline Zhang and Dumitrica (2008) & TB & 0.34 & 1.26 & 0.455 & 0.38 \\
\hline Gupta and Batra (2008) & Molecular mechanics & 0.34 & $0.93-1$ & $0.38-0.44$ & 0.19 \\
\hline \multicolumn{6}{|c|}{ Experimental studies } \\
\hline Krishnan et al. (1998) & Thermal vibrations & 0.34 & $1.3 \pm 0.4$ & - & - \\
\hline Salvetat et al. (1999) & AFM & 0.34 & 1 & - & - \\
\hline
\end{tabular}

TABLE 2: Young's modulus, shear modulus, and Poisson's ratio values for graphene

\begin{tabular}{|l|l|l|l|l|}
\hline Researchers & $\begin{array}{l}\text { Thickness } \\
(\mathbf{n m})\end{array}$ & $\begin{array}{l}\text { Young's modulus } \\
\text { (TPa) }\end{array}$ & $\begin{array}{l}\text { Shear modulus } \\
\text { (TPa) }\end{array}$ & Poisson's ratio \\
\hline Lu (1997) & 0.34 & 1.02 & - & 0.16 \\
\hline Van Lier et al. (2000) & 0.34 & 1.11 & - & - \\
\hline Chang et al. (2005) & 0.34 & 1.06 & - & 0.16 \\
\hline Caillerie et al. (2006) & 0.34 & 0.82 & - & 0.26 \\
\hline Huang et al. (2006) & 0.0678 & 3.58 & - & 0.397 \\
\hline Reddy et al. (2006) & 0.34 & 1.11 & - & 0.25 \\
\hline Wu et al. (2006) & 0.258 & 1.06 & 0.418 & 0.27 \\
\hline Meo and Rossi (2006) & 0.34 & 0.945 & - & - \\
\hline
\end{tabular}

\subsection{Modeling of the Atomic Interactions}

As mentioned earlier, CNT carbon atoms are bonded together with covalent bonds forming a hexagonal lattice. These bonds have a characteristic length and direction angle expressed in a 3D basis. Therefore, the total deformation of 
the nanotube is the result of the interactions between these bonds. As previously performed in Qian et al. (2002), the atomic interactions are described using a relation between molecular and structural mechanics. Thus, the nanotubes are assumed to behave as space frame structures (Li and Chou, 2003).

From the viewpoint of molecular mechanics, CNTs may be regarded as large molecules consisting of carbon atoms. The general expression of the total potential energy is the following sum of energies due to the valence of bonded interactions and nonbonded interactions (Rappe et al., 1992):

$$
U_{\text {total }}=\sum U_{r}+\sum U_{\theta}+\sum U_{\Phi}+\sum U_{w}+\sum U_{V d W}
$$

where $U_{\tau}$ is the energy due to bond stretch interaction, $U_{\theta}$ is the energy due to bending bond angle variations, $U_{\phi}$ is the energy due to the dihedral angle torsion, $U_{w}$ is the energy due to out-of-plane torsion, and $U_{V d W}$ is the energy due to nonbonded van der Waals interactions.

For the covalent bond, the main contributions to the total energy come from the first four terms of Eq. (4.1) (Rappe et al., 1992). Moreover, under the assumption of small deformation, the harmonic approximation is adequate for describing the energy (Liu et al., 2006). By adopting the simplest harmonic forms and merging dihedral angle torsion and out-of-plane torsion into a single equivalent term, we get for each energy term

$$
\left\{\begin{array}{l}
U_{r}=\frac{1}{2} k_{r}\left(r-r_{0}\right)^{2}=\frac{1}{2} k_{r}(\Delta r)^{2} \\
U_{\theta}=\frac{1}{2} k_{\theta}\left(\alpha-\alpha_{0}\right)^{2}=\frac{1}{2} k_{\theta}(\Delta \alpha)^{2} \\
U_{\tau}=U_{\phi}+U_{w}=\frac{1}{2} k_{\tau}(\Delta \phi)^{2}
\end{array},\right.
$$

where $k_{r}, k_{\theta}$, and $k_{\tau}$ are the bond-stretching force constant, bond angle-bending force constant, and torsional resistance, respectively, and the symbols $\Delta r, \Delta \alpha$, and $\Delta \phi$ represent the increment of the bond stretching, the bond angle, and the angle of bond twisting, respectively. It is important to notice that these $k_{i}$ parameters are actually constant because of the choice of harmonic energies.

The interactions between the atoms are modeled using Bernoulli's 3D linear beams, whose stiffness is defined in such a way that the beam strain energy is equal to the potential energy due to atomic interactions. The length of the beam corresponds to the interatomic distance $\alpha_{C-C}$, i.e., $0.1421 \mathrm{~nm}$. According to the theory of classical structural mechanics ( $\mathrm{Li}$ and Chou, 2003), a direct relationship between the structural mechanics parameters, the tensile resistance $E A$, the flexural rigidity $E I$, the torsional stiffness $G J$, and the molecular mechanics parameters $k_{r}, k_{\theta}$, and $k_{\tau}$ is deduced as follows:

$$
\frac{E A}{\alpha_{C-C}}=k_{r}, \quad \frac{E I}{\alpha_{C-C}}=k_{\theta}, \quad \text { and } \quad \frac{G J}{\alpha_{C-C}}=k_{\tau} .
$$

In the present model, for the $k_{r}, k_{\theta}$, and $k_{\tau}$ constants, the values of $6.52 \times 10^{-7} \mathrm{~N} \mathrm{~nm}^{-1}, 8.76 \times 10^{-10} \mathrm{~N} \mathrm{~nm} \mathrm{rad}^{-2}$, and $2.78 \times 10^{-10} \mathrm{~N} \mathrm{~nm} \mathrm{rad}^{-2}$ taken from Cornell et al. (1995) have been, respectively, adopted.

Since CNTs may be represented as a lattice structure with a linear elastic behavior and a regular microstructure, the objective is now the description of the homogenization process in order to obtain their effective properties.

\subsection{Homogenization Theory}

The starting point of the homogenization theory is the 3D elastic problem posed on the nanotube which is modeled as a space frame structure. The nanotube is a slender structure and has a periodic structure along its axis. Therefore, the elastic problem exhibits two small parameters: $e$, which corresponds to the slenderness of the structure, and $\varepsilon$, which represents the ratio of the length of the axial period to the total length of the structure. These two parameters are of the same order of magnitude and tend to zero simultaneously, thus allowing use of the asymptotic expansion method 
with one small parameter (Buannic and Cartraud, 2001). The displacement solution of the elastic problem is searched as follows:

$$
u(x)=u_{\alpha}^{0}\left(x_{3}\right) e_{\alpha}+\varepsilon u^{1}\left(x_{3}, y_{1}, y_{2}, y_{3}\right)+\varepsilon^{2} u^{2}\left(x_{3}, y_{1}, y_{2}, y_{3}\right)+\ldots,
$$

where $x_{i}$ are the macroscopic variables of the problem related to the global axis system $\left(e_{1} ; e_{2} ; e_{3}\right)$ (see Fig. 5). The microscopic variables are defined as follows:

$$
y_{i}=x_{i} / \varepsilon .
$$

The functions $u^{k}\left(x_{3}, y_{1}, y_{2}, y_{3}\right)$ are periodic in variable $y_{3}$ (the length of the cell $Y$ at the microscopic scale, see Fig. 5), which are denoted $Y_{3}$ periodic in the following equation:

$$
u^{k}\left(x_{3}, y_{1}, y_{2}, y_{3}\right)=u^{k}\left(x_{3}, y_{1}, y_{2}, y_{3}+Y_{3}\right) .
$$

In Eqs. (4.4), (4.5), and (4.6), the 3D elasticity problem splits into a sequence of 3D microscopic problems, posed on the axial basic cell, and 1D macroscopic problems, providing the overall nanotube response. Only the main results of the method are reported here; for more details see Buannic and Cartraud (2001).

The solution of the leading order (first-order) microscopic elastic problem is expressed by

$$
u^{1}=-y_{\alpha} \partial_{3} u_{\alpha}^{0}\left(z_{3}\right) \mathbf{e}_{3}+\hat{u}^{1}\left(z_{3}\right)+\varphi^{1}\left(z_{3}\right)\left(y_{1} \mathbf{e}_{2}-y_{2} \mathbf{e}_{1}\right),
$$

where $\partial_{3}$ denotes $\partial / \partial x_{3}, \hat{u}_{3}^{1}$ and $\varphi^{1}$ are the macroscopic axial displacement and rotation, and $\hat{u}_{\alpha}^{0}$ is the transverse displacements (see Fig. 4).

From the previous solution, the data of the next order (0th order) of microscopic problem are the macroscopic strain components of extension $\left(E^{E}\right)$, curvatures $\left(E^{C_{1}}\right.$ and $\left.E^{C_{2}}\right)$, and torsion $\left(E^{T}\right)$, which are given by $\left(\partial_{3.3}\right.$ denoting $\left.\partial^{2} / \partial x_{3}^{2}\right)$

$$
\left\{\begin{array}{rl}
E^{E}\left(x_{3}\right) & =\partial_{3} \hat{u}_{3}^{1}\left(x_{3}\right) \\
E^{C_{1}}\left(x_{3}\right) & =\partial_{33} u_{1}^{0}\left(x_{3}\right) \\
E^{C_{2}}\left(x_{3}\right) & =\partial_{33} u_{2}^{0}\left(x_{3}\right) \\
E^{T}\left(x_{3}\right) & =\partial_{3} \varphi^{1}\left(x_{3}\right)
\end{array} .\right.
$$

The leading-order macroscopic problem is obtained from the compatibility conditions of the first-order microscopic problem. This problem generalizes and justifies the Euler-Bernoulli-Navier's model. The macroscopic behavior which is involved in this beam problem is obtained from the solution of the previous 0th-order microscopic problem, which we call the basic cell problem.
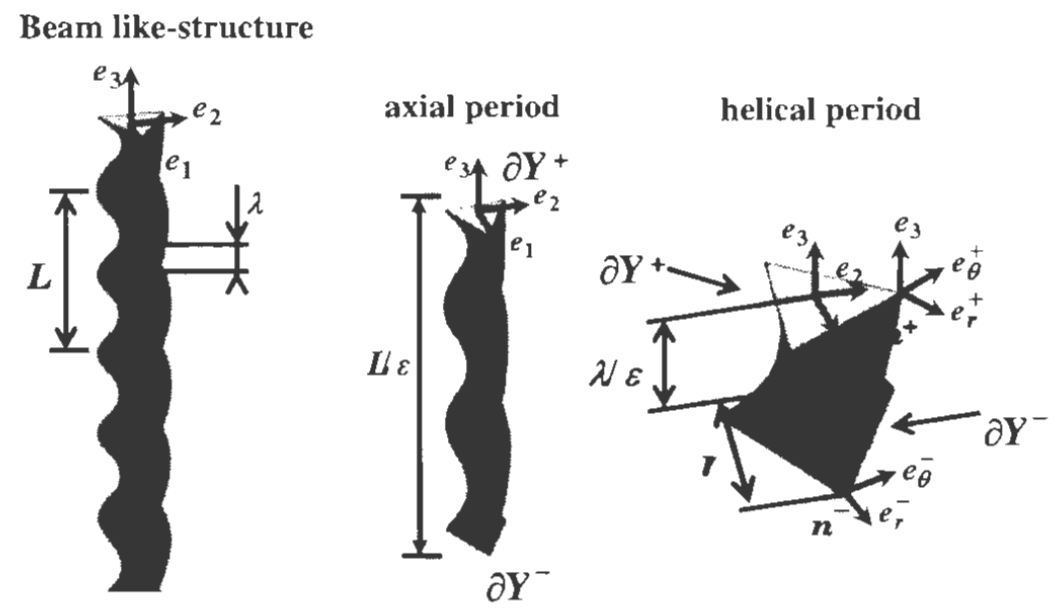

FIG. 5: Periodic helical structure and the corresponding basic cell 
The numerical implementation of the basic cell problems using FE software appears to be very easy. The data corresponding to a macroscopic strain are imposed through a set of linear relationships relating displacement components of the opposite nodes (situated on the + and - sides) (Cartraud and Messager, 2006; Messager and Cartraud, 2008):

$$
\left\{\begin{array}{l}
U_{1}^{+}-U_{1}^{-}=Y_{3}\left(\bar{y}_{3} E^{C_{1}}-y_{2} E^{T}\right) \\
U_{2}^{+}-U_{2}^{-}=Y_{3}\left(\bar{y}_{3} E^{C_{2}}-y_{1} E^{T}\right) \\
U_{3}^{+}-U_{3}^{-}=Y_{3}\left(E^{E}-y_{1} E^{C_{1}}-y_{2} E^{C_{2}}\right) \\
\Theta_{1}^{+}-\Theta_{1}^{-}=Y_{3} E^{C_{1}} \\
\Theta_{2}^{+}-\Theta_{2}^{-}=Y_{3} E^{C_{2}} \\
\Theta_{3}^{+}-\Theta_{3}^{-}=Y_{3} E^{T}
\end{array}\right.
$$

where $\bar{y}_{3}=\left(y_{3}^{+}+y_{3}^{-}\right) / 2, y_{\alpha}=y_{\alpha}^{+}=y_{\alpha}^{-}$, and where the superscript - and + denotes the two parts $\partial Y^{-}$and $\partial Y_{\text {, }}^{+}$ respectively, of the period boundary concerned with periodicity conditions (see Fig. 5).

Considering the expression of the total strain energy,

$$
\Pi=\frac{1}{2} \int_{\lambda / \varepsilon} \sigma: e d y=\frac{Y_{3}}{2}\left\{\begin{array}{c}
F \\
M_{1} \\
M_{2} \\
M_{3}
\end{array}\right\}^{T}\left\{\begin{array}{c}
E^{E} \\
E^{C_{1}} \\
E^{C_{2}} \\
E^{T}
\end{array}\right\}=\frac{Y_{3}}{2}\left\{\begin{array}{c}
E^{E} \\
E^{C_{1}} \\
E^{C_{2}} \\
E^{T}
\end{array}\right\}\left[K^{\text {hom }}\right]\left\{\begin{array}{c}
E^{E} \\
E^{C_{1}} \\
E^{C_{2}} \\
E^{T}
\end{array}\right\},
$$

the homogenized stiffness coefficients $K_{i j}^{\text {hom }}$ can be deducted afterward from different combination sets of strain components. $K_{E E}^{\text {hom }}$ is thus obtained by applying $E^{E}=1$ and $E^{C_{1}}=E^{C_{2}}=E^{T}=0 ; K_{C_{1} C_{1}}^{\text {hom }}$ is obtained by applying $E^{C_{1}}=1$ and $E^{E}=E^{C_{2}}=E^{T}=0$, etc.

The homogenized beam behavior of the nanotube (i.e., the matrix $\left[K^{\text {hom }}\right]$ coefficients) can thus be computed from the solution of these basic cell problems posed on its axial period. The geometrical description of this axial period is given in the Appendix.

The homogenization theory can be seen as an optimal method to derive the effective nanotube properties. Instead of considering a large part of the nanotube submitted to mechanical tests, the analysis actually involves only an axial period. This process can be applied one step further, in the case where the loadings of the basic cell problem fulfill helical symmetry, which is encountered for traction and torsion. Thus it is possible to define a reduced helical basic cell as depicted in Fig. 5.

In the case of SWCNT, the periodicity in the plane of the graphene sheet, characterized by the two translation vectors $\vec{a}_{M}$ and $\vec{a}_{M}-\vec{a}_{N}$, leads to two helical symmetry properties for the nanotube. The definition of a reduced unit cell with the aim to minimize the computational domain has been addressed in several papers (e.g., Barros et al., 2006; Dumitrica and James, 2007; Zhang and Dumitrica, 2008), where it is shown that a two-atom reduced unit cell can be used in the case of an atomistic framework. Since in this work interatomic interactions are modeled with a structural mechanics approach, the basic cell is composed of three interatomic bonds.

The definition of our helical basic cell is shown in Fig. 6, associating two couples of opposite nodes $\left(\mathrm{m}^{+}, \mathrm{m}^{-}\right)$ and $\left(n^{+}, n^{-}\right)$on a half-hexagon. From this helical basic cell, $\lambda / \varepsilon$ denoting its axial length, the initial periodicity property defined in Eq. (4.5) now becomes

$$
u^{k}\left(r, \theta^{-}, y_{3}\right) \times e_{i}^{-}=u^{k}\left(r, \theta^{-}+\frac{2 \pi L}{\lambda}, y_{3}+\frac{\lambda}{\varepsilon}\right) \times e_{i}^{+},
$$

then expressed in the local cylindrical coordinates $(i=r, \theta, 3)$ (Messager and Cartraud, 2008). Thus, one can define a problem posed on this helical basic cell of length $\lambda / \varepsilon$ to compute the axial properties of an SWCNT. Denoting $U_{i}^{-}$(as typed above) and $U_{i}^{+}$the degrees of freedom expressed in the local cylindrical frames $\left(e_{r}^{-} ; e_{\theta}^{-} ; e_{3}^{+}\right)$and $\left(e_{r}^{+} ; e_{\theta}^{+} ; e_{3}^{+}\right)$ of the opposite nodes $\left(m^{+}, m^{-}\right)$and $\left(n^{+}, n^{-}\right)$of the FE model (see Fig. 6), the linear relationships corresponding to a given axial macroscopic strain are 


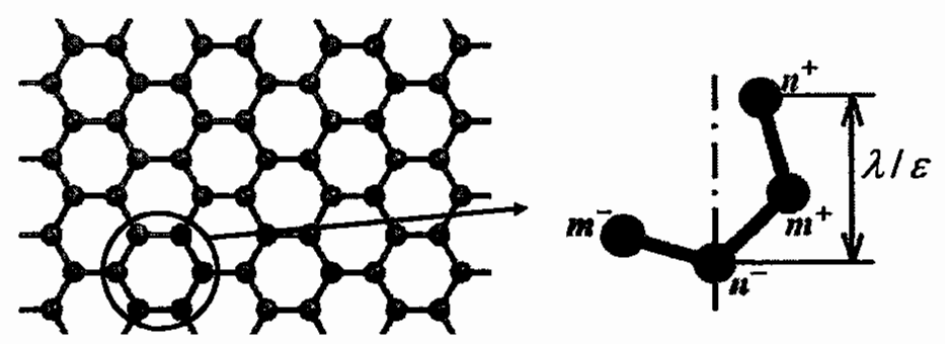

FIG. 6: Helical basic cell of an SWCNT

$$
\left\{\begin{array}{l}
U_{r}^{+}-U_{r}^{-}=0 \\
U_{\theta}^{+}-U_{\theta}^{-}=r \lambda E^{T} / \varepsilon \\
U_{3}^{+}-U_{3}^{-}=\lambda E^{E} / \varepsilon \\
\Theta_{r}^{+}-\Theta_{r}^{-}=0 \\
\Theta_{\theta}^{+}-\Theta_{\theta}^{-}=0 \\
\Theta_{3}^{+}-\Theta_{3}^{-}=\lambda E^{T} / \varepsilon
\end{array} .\right.
$$

These relationships are expressed in the local cylindrical frames represented in Fig. 5. It is possible to check that these relations derived from the helical symmetry properties expressed in Eq. (4.11) are consistent with the objective molecular dynamic formulation (Dumitrica and James, 2007; Zhang and Dumitrica, 2008). The strain energy is given by

$$
\Pi=\frac{1}{2} \int_{\lambda / \varepsilon} \sigma: e d y=\frac{\lambda}{2 \varepsilon}\left\{\begin{array}{c}
F \\
M
\end{array}\right\}^{T}\left\{\begin{array}{l}
E^{E} \\
E^{T}
\end{array}\right\}=\frac{\lambda}{2 \varepsilon}\left\{\begin{array}{c}
E^{E} \\
E^{T}
\end{array}\right\}^{T}\left[\begin{array}{cc}
K_{E E}^{\text {hom }} & K_{E T}^{\text {hom }} \\
K_{T E}^{\text {hom }} & K_{T T}^{\text {hom }}
\end{array}\right]\left\{\begin{array}{c}
E^{E} \\
E^{T}
\end{array}\right\},
$$

and is used to compute the axial homogenized behavior of the nanotube.

\section{NUMERICAL RESULTS AND DISCUSSION}

\subsection{Stiffness of a Graphene Sheet}

In the first step, the overall properties of a graphene sheet are calculated. The aim is twofold. It first enables us to compare the overall material properties identified from the homogenized beam properties of the nanotube to those of the graphene sheet. It also allows validation of the homogenization approach used for the nanotube, since it is expected that for large diameters, the overall material properties of the nanotube tend to be similar to those of the graphene sheet.

In this section, the effective properties of the graphene sheet correspond to an overall plane stress behavior. Therefore, the classical homogenization theory for periodic media is used (Bendsoe, 1989), the macroscopic strain being a constant in the basic cell.

The periodic basic cell of the plane graphene sheet can be obtained from the repetition of a half of a hexagon, using the two translation vectors $\vec{a}_{M}$ and $\vec{a}_{M}-\vec{a}_{N}$. These vectors actually allow switching from the carbon atom denoted $n_{1}^{-}$to the carbon atom $n_{1}^{+}$, and in the same way allow switching for the couple $n_{2}^{-}$and $n_{2}^{+}$atoms, as shown in Fig. 6.

Therefore, the basic cell reduces to these four atoms, which are represented by nodes in the finite element model with Bernoulli's beam element between them, according to the structural mechanics modeling approach described in Section 4.2. 
The effective properties of the graphene sheet are deducted from the solution of the $2 \mathrm{D}$ basic cell problem. The data of this problem is a macroscopic strain, with three components denoted $E^{11}, E^{22}$, and $E^{12}$ associated to the extensions and shear in the plane, respectively. The corresponding deformed shapes are presented in Fig. 7.

The overall strain energy $\Pi$ of the graphene is given by

$$
\frac{2 \Pi}{S_{\text {cell }}}=\left\{\begin{array}{lll}
E^{11} & E^{22} & 2 E^{12}
\end{array}\right\}\left[a^{\text {hom }}\right]\left\{\begin{array}{c}
E^{11} \\
E^{22} \\
2 E^{12}
\end{array}\right\},
$$

where $S_{\text {cell }}$ is the basic cell surface and $\left[a^{\text {hom }}\right]$ is the matrix of coefficients stiffness in plane stress:

$$
\left[a^{\text {hom }}\right]=\left[\begin{array}{lll}
a_{11} & a_{12} & a_{13} \\
a_{21} & a_{22} & a_{23} \\
a_{31} & a_{32} & a_{33}
\end{array}\right]
$$

Based on classical properties of homogenization theory of periodic media, e.g., Léné (1984), this matrix is symmetric. Moreover, due to the hexagonal symmetry of the basic cell, it is shown that the 2D homogenized behavior is isotropic (Léné, 1984).

Using this method, the numerical results for $\left[a^{\text {hom }}\right]$ are in very good agreement with the isotropy property of the overall behavior; the terms $\left(a_{13}, a_{23}, a_{31}, a_{32}\right)$ of the matrix are actually negligible compared to the others, and the relation $2 a_{33}=a_{11}-a_{22}$ is fulfilled by the numerical results. From these results, and with the thickness $t$ taken as the interlayer spacing of the graphene sheet, i.e., $0.34 \mathrm{~nm}$ [see Dresselhauss et al. (2001)], the homogenized material properties of the graphene sheet are found to be $E_{\text {graphite }}=1.0482 \mathrm{TPa}, G_{\text {graphite }}=0.49 \mathrm{TPa}$, and $v_{\text {graphite }}=0.06$.

As can be seen from Table 2, several authors have calculated the Young's modulus of graphene sheet to be around $1 \mathrm{TPa}$ for a wall thickness equal to $0.34 \mathrm{~nm}$. The Young's modulus of a graphene sheet has been computed using an ab initio method by Van Lier et al. (2000) as $1.11 \mathrm{TPa}$. Using a continuum mechanics approach based on Brenner potential, Reddy et al. (2006) calculated the Young's modulus of graphene to be around 1.11 TPa and the Poisson ratio to be around 0.25 . Caillerie et al. (2006), using a discrete homogenization technique, calculated a Young's modulus and Poisson ratio for graphene sheet equal to $0.82 \mathrm{TPa}$ and 0.26 , respectively. From TB simulations, Hernandez et al. (1998) calculated the Young's modulus of graphite to be about $1.02 \mathrm{TPa}$. Chang et al. (2005) gave graphite a Young's modulus value of $1.06 \mathrm{TPa}$ using an analytical molecular structure mechanics model. Using the modified Morse potential, Meo and Rossi (2006) calculated the Young's modulus of graphene sheet to be around $0.945 \mathrm{TPa}$.

The results for the Young's and shear moduli presented in our study are consistent with the data reported in the literature (see Table 2). However, the results are different for the Poisson ratio - the value obtained in this work is lower than the Poisson ratio calculated by Caillerie et al. (2006), Reddy et al. (2006), and Huang et al. (2006). The difference between the results of the present work and those coming from other numerical studies results from the use of different potentials.

\subsection{Overall Axial Stiffness of SWCNTs}

In this section, the homogenization theory described in Section 4.2 is applied for the determination of the axial behavior of SWCNTs. In this case, the helical symmetry property can be taken into account, and the traction and

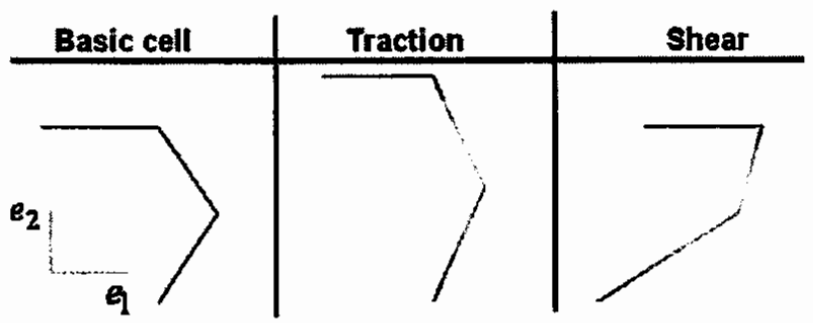

FIG. 7: Computations of the elementary plane cells 
torsion homogenized properties are computed, i.e., three terms of the symmetric matrix in Eq. (3.1): $K_{E E}^{\text {hom }}, K_{T T}^{\text {hom }}$, and $K_{E T}^{\text {hom }}=K_{E T}^{\text {hom }}$.

In the following, zigzag, armchair, and SWCNTs or arbitrary chirality are considered. Thanks to helical symmetry, the basic cell reduces to four atoms only, as depicted in Fig. 8, but contrary to the case of the graphene sheet, the basic cell is not plane. The homogenization computations are performed from the linear relationships of Eq. (4.12).

First, the homogenization theory is validated by comparison with the reference results obtained in Tserpes and Papanikos (2005). In this reference the homogenized properties of the nanotube are computed from the analysis of a nanotube which includes a large number of atoms. Along with the results given in Tserpes and Papanikos (2005), mechanical tests are also performed in our study on a macroscopic finite element model [see Fig. 8(a)] solved with the commercial code Samcef. The macroscopic properties of the nanotube are identified from its response. The results given in Table 3 for the axial stiffness show that our results are in very good agreement with those reported in this reference for three different nanotube geometries: $(8,8),(14,0)$, and $(11,5)$. The results for Samcef large FE models are also given. The homogenized stiffness in torsion can also be validated using the same approach [see Messager and Cartraud (2008)].

Next, the study of the tensile, torsion, and the coupling coefficients stiffness evolutions of $(M, 0)$ zigzag, $(M, M)$ armchair, and $(M, N)$ chiral SWCNTs was performed from the solution of the helical basic cell problem. The corresponding variations of axial stiffness components with regard to the nanotube diameter $D$ are presented in Figs. 9(a) and $9(\mathrm{~b})$. The tensile stiffness is proportional to $D$, and the torsion coefficient evolutions are found to follow nearly cubic dependences on $D$. Furthermore, the tensile and torsion coefficients' stiffnesses depend weakly on the chiral angle. Moreover, the coupling coefficients for zigzag and armchair nanostructures logically vanish due to the axial symmetry, i.e., $K_{E T}^{\text {hom }}=K_{T E}^{\text {hom }}=0$. For chiral SWCNTs, the results given in Fig. 9 correspond to $M \geq N$, which yields a chiral angle $0 \leq \theta \leq 30^{\circ}$. In this range, the coupling coefficients stiffness is maximum for $\theta=15^{\circ}$, as shown in Fig. 9(c). Moreover, it has been checked that $K_{E T}^{\text {hom }}(\theta)=-K_{E T}^{\text {hom }}(-\theta)$.

\subsection{Bending Stiffness of SWCNTs}

In order to complete the determination of the homogenized beam properties of the SWCNTs [see Eq. (3.1)], its bending behavior has to be considered. In that case, due to the loading mode discussed in Section 4.2, the helical symmetry

TABLE 3: Tensile stiffness coefficients of $(8,8),(14,0)$, and $(11,5)$ SWCNTs

\begin{tabular}{|c|c|c|c|}
\hline & \multicolumn{3}{|c|}{$K_{E E}^{\text {hom }}\left(10^{-6} N\right)$} \\
\hline & $\mathbf{( 8 , 8 )}$ & $(\mathbf{1 4 , 0 )}$ & $(\mathbf{1 1 , 5})$ \\
\hline Microscopic FE & 1.216 & 1.215 & 1.239 \\
\hline Macroscopic FE & 1.216 & 1.215 & 1.24 \\
\hline Macroscopic FE (Tserpes and Papanikos, 2005) & 1.217 & 1.215 & 1.24 \\
\hline
\end{tabular}

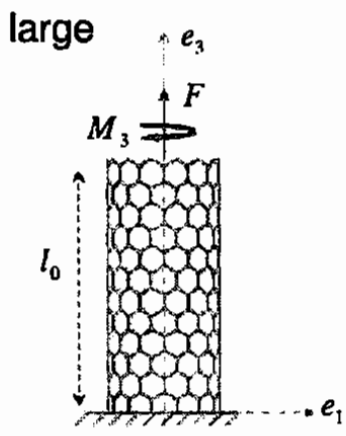

microscopic

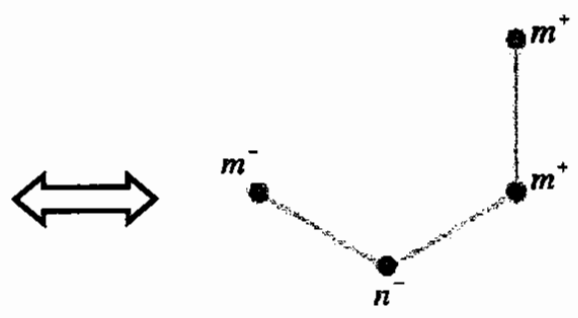

FIG. 8: Present FE models: (a) macroscopic model of the SWCNT $(14,0)$ and (b) helical basic cell. 


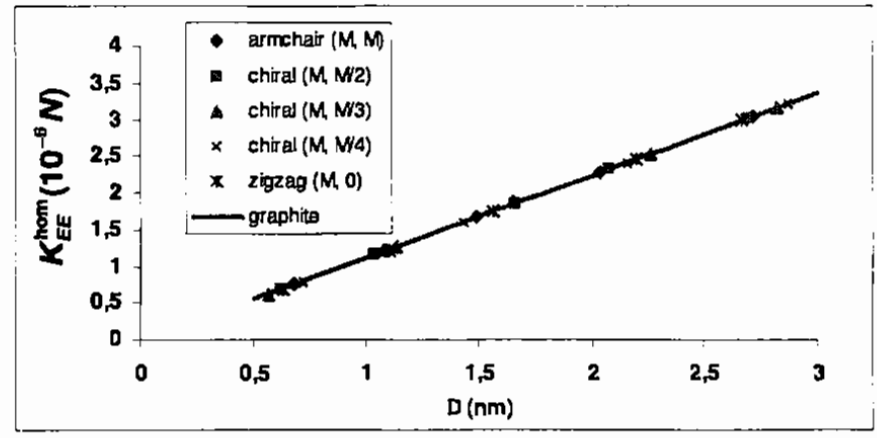

(a)

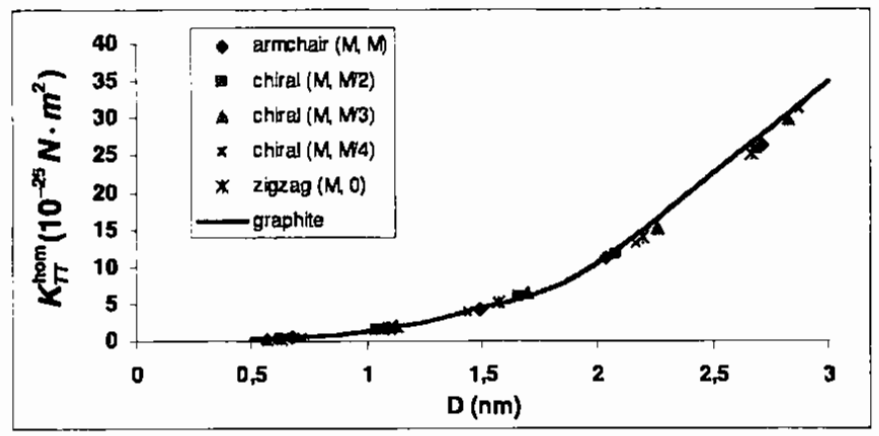

(b)

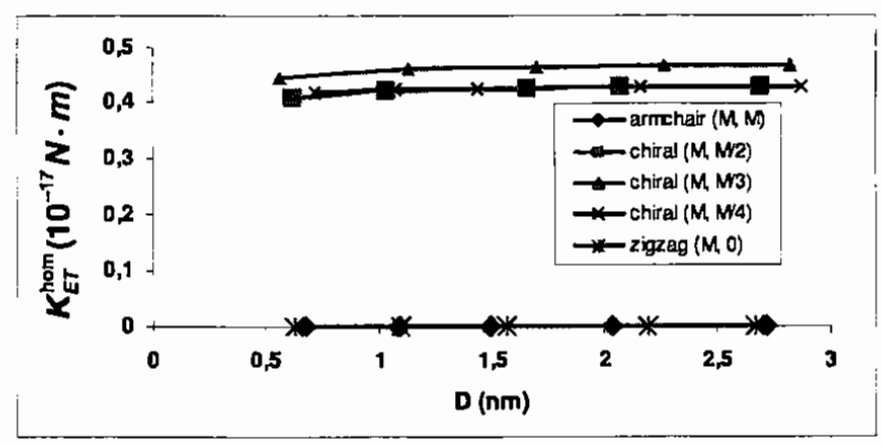

(c)

FIG. 9: Traction (a), torsion (b), and coupling traction-torsion (c) stiffness evolutions of zigzag, armchair, and chiral SWCNTs.

properties cannot be used. Thus, the basic cell is defined from the axial periodicity property of the nanotube. The geometric description of this axial period is given in the Appendix.

The FE model of this cell was generated thanks to a Matlab macro developed for armchair, zigzag, and chiral nanotubes. In the following, the chiral indices were chosen of the form (M, M/2), (M, M/3), and (M, $M / 4)$. The case $(10,5)$ is presented in Fig. 10(a). From the axial basic cell, the homogenization theory described in Section 4.2 is implemented, which consists of linear relationships between two opposite nodes of the basic cell [see Eq. (4.8)]. Since the traction-torsion properties are already known from the results of the previous section, seven new computations need to be performed to obtain the whole symmetric four-by-four matrix given in Eq. (3.1). The deformed shape obtained for a macroscopic curvature $E^{C_{2}}$ is presented in Fig. 10(b).

Similar to the overall axial behavior, the results for the bending stiffnesses were checked by comparison with the macroscopic FE model response solved with Samcef. Based on the results of the homogenization problems, it is

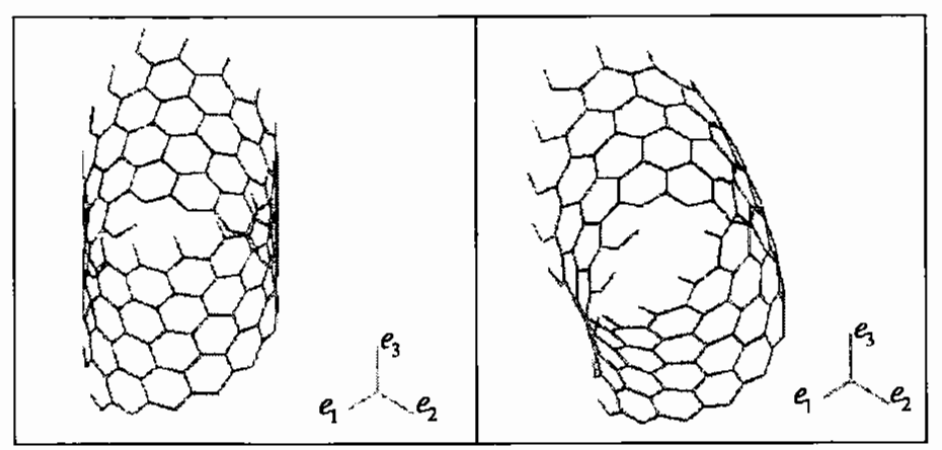

FIG. 10: Axial basic cell for chiral nanotube $(10,5)$ (a) in the initial configuration and (b) submitted to a macroscopic curvature $E^{C_{2}}$. 
found that in all cases the bending behavior is uncoupled from traction and torsion. Moreover, the bending behavior is isotropic, since $K_{C_{1} C_{1}}^{\mathrm{hom}}=K_{C_{2} C_{2}}^{\mathrm{hom}}$ and $K_{C_{1} C_{2}}^{\mathrm{hom}}=0$. The latter result is in agreement with those of the macroscopic model, from which the curvature of the nanotube is independent of the bending moment direction.

Thus, the homogenized SWCNT beam behavior is of the form

$$
\left[K^{\text {hom }}\right]=\left[\begin{array}{cccc}
K_{E E}^{\text {hom }} & 0 & 0 & K_{E T}^{\text {hom }} \\
& K_{B B}^{\text {hom }} & 0 & 0 \\
& & K_{B B}^{\text {hom }} & 0 \\
+ \text { sym. } & & & K_{T T}^{\text {hom }}
\end{array}\right]
$$

The bending stiffness evolutions of $(M, 0)$ zigzag, $(M, M)$ armchair, and $(M, N)$ chiral SWCNTs for increasing nanotube diameters were then studied. The variation of $K_{B B}^{\text {hom }}$ with regard to nanotube diameter $D$ is presented in Fig. 11. The bending coefficient evolutions follow nearly cubic dependences on $D$ and are weakly dependent on the chiral angle.

The results of the analysis of the overall mechanical behaviors of different nanotubes are summarized in Table 4.

TABLE 4: Overall mechanical behavior of SWCNTs

\begin{tabular}{|c|c|c|l|c|c|c|}
\hline & Tube & $\begin{array}{c}D_{\text {exact }} \\
(n m)\end{array}$ & $\begin{array}{l}K_{E E}^{\text {hom }} \\
\left(10^{-6} N\right)\end{array}$ & $\begin{array}{c}K_{T T}^{\text {hom }} \\
\left(10^{-25} N . m^{2}\right)\end{array}$ & $\begin{array}{c}K_{E T}^{\text {hom }} \\
\left(10^{-17} N . m\right)\end{array}$ & $\begin{array}{c}K_{B B}^{\text {hom }} \\
\left(10^{-24} N . m^{2}\right)\end{array}$ \\
\hline Zigzag & $(5,0)$ & 0.3982 & 0.402909 & 0.080825 & 0 & 0.007987 \\
\hline & $(8,0)$ & 0.63079 & 0.67876 & 0.328 & 0 & 0.032859 \\
\hline & $(15,0)$ & 1.1773 & 1.3039 & 2.1524 & 0 & 0.224221 \\
\hline & $(20,0)$ & 1.56848 & 1.74594 & 5.0979 & 0 & 0.534664 \\
\hline Armchair & $(5,5)$ & 0.68222 & 0.76017 & 0.380353 & 0 & 0.044681 \\
\hline & $(8,8)$ & 1.08789 & 1.21628 & 1.63858 & 0 & 0.180665 \\
\hline & $(14,14)$ & 1.901066 & 2.12849 & 8.97866 & 0 & 0.962832 \\
\hline & $(20,20)$ & 2.71484 & 3.04071 & 26.321733 & 0 & 2.803289 \\
\hline Chiral & $(6,3)$ & 0.62592 & 0.68997 & 0.297449 & 0.004065 & 0.033803 \\
\hline & $(8,4)$ & 0.83217 & 0.92378 & 0.725923 & 0.004157 & 0.079965 \\
\hline & $(14,7)$ & 1.45269 & 1.62265 & 3.99252 & 0.004240 & 0.428103 \\
\hline & $(20,10)$ & 2.074 & 2.32026 & 11.71585 & 0.004261 & 1.247674 \\
\hline
\end{tabular}

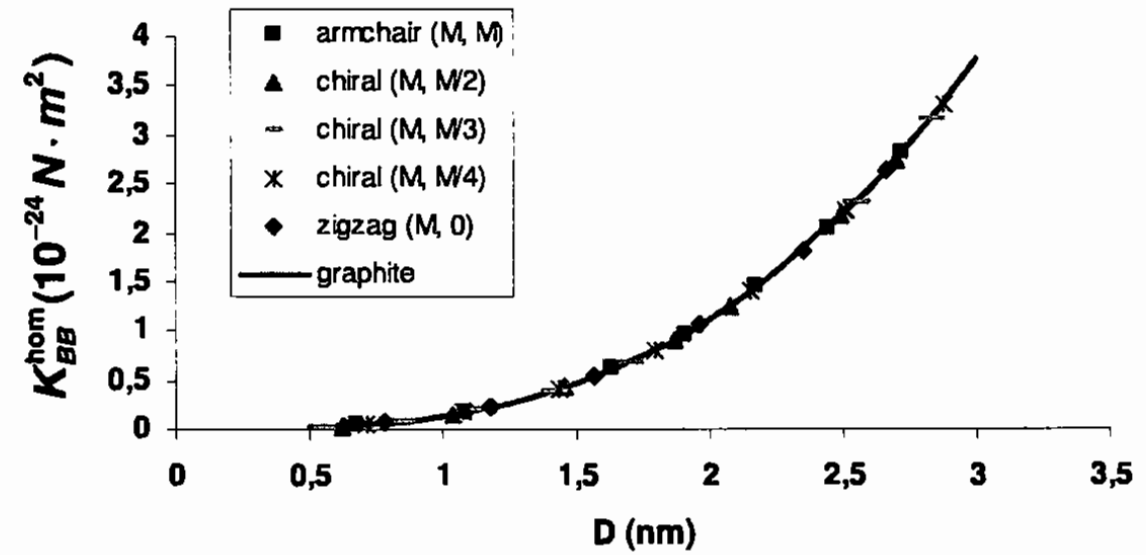

FIG. 11: Bending stiffness evolutions of zigzag, armchair, and chiral SWCNTs 


\subsection{Equivalent Young's Modulus of SWCNTs}

Once the overall beam behavior of the nanotube has been obtained, its equivalent Young's modulus can be defined from the traction or bending stiffness, the bending behavior being isotropic [see Eq. (5.3)].

If the stiffness in traction is used, i.e., the first term on the diagonal in Eq. (5.3), the Young's modulus is given by Eq. (3.2) with $A_{0}=\pi \cdot D_{\text {exact }} . t$, hence:

$$
E_{S W C N T}^{T}=\frac{K_{E E}^{\text {hom }}}{\pi D_{\text {exact }} t} .
$$

Otherwise, from the bending stiffness-second or third term on the diagonal-the Young's modulus is provided by the ratio of this stiffness to the moment of inertia of the cross-sectional area. Using for this moment of inertia the expression proposed in Wang and Varadan (2005), one obtains

$$
E_{S W C N T}^{B}=\frac{K_{B B}^{\text {hom }}}{\pi\left(\frac{D_{\text {exact }}}{2}\right)^{3} t} .
$$

In Eqs. (5.4) and (5.5), the geometrical properties are calculated with the approximation $t \ll D_{\text {exact }}$. However, the true justification of these expressions is different. Our aim is the comparison of the SWCNT material properties with the graphene 2D plane-stress homogenized behavior, which are proportional to the sheet thickness $t$. Therefore, as the value of this thickness is not well established, it is important to use expressions which are also proportional to $t$ for the geometric properties of the SWCNT cross section. In the same way, the value of the thickness is the same as the one used in Section 5.1, i.e., $t=0.34 \mathrm{~nm}$.

Next, the scale effects for SWCNTs are studied through the evolution of traction and through bending Young's moduli as a function of the exact diameter. The results are shown in Fig. 12. To perform comparisons, the graphene equivalent modulus value (obtained in Section 5.1) has also been displayed. As seen in Fig. 12, for both the traction and bending Young's moduli, as the diameter increases, their values converge to the homogenized Young's modulus of the graphene sheet, i.e., $1.048 \mathrm{TPa}$ (see Section 5.1). The nanotube traction Young's modulus is very close to the homogenized value of the graphene sheet, the difference being less than 5\% [see Fig. 12(a)]. For the nanotube bending Young's modulus, these differences reach $10 \%$ for diameters between 0.5 and $1 \mathrm{~nm}$ but are less than $3 \%$ if the diameter is greater than $1 \mathrm{~nm}$.

It can also be seen that for a given diameter, the traction and bending Young's moduli are maximum for the armchair configuration and minimum for the zigzag one. The same results were obtained in Kalamkarov et al. (2006),

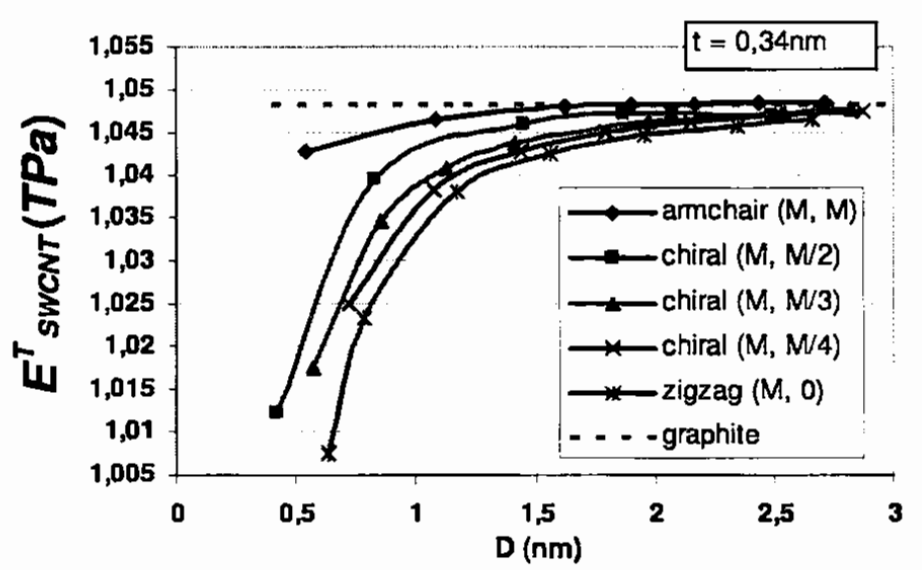

(a)

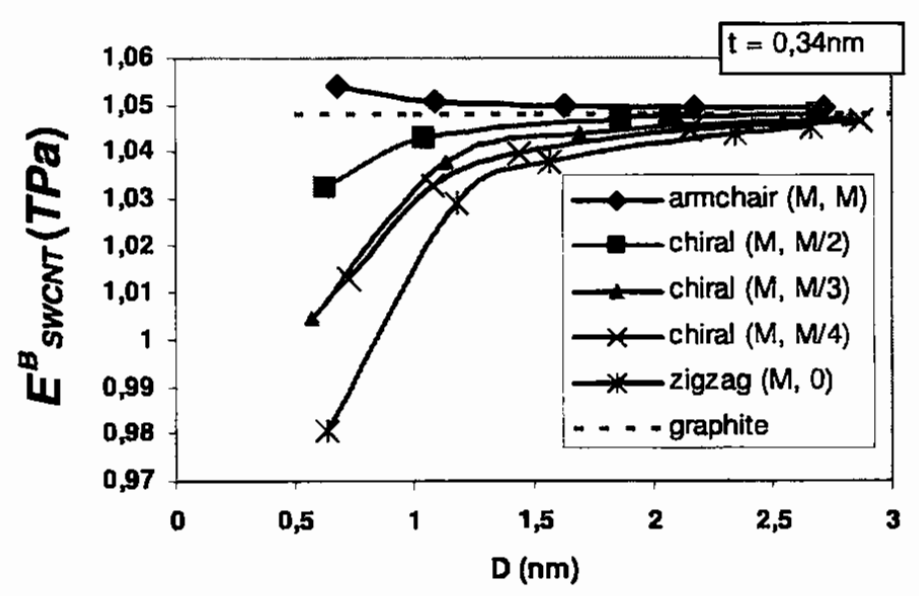

(b)

FIG. 12: Variation of Young's modulus of different types of SWCNTs with tube diameter compared to the graphite value (a) from the traction stiffness and (b) from the bending stiffness. 
Giannopoulos et al. (2008), Wu et al. (2006), Chang et al. (2005), Li and Chou (2003), and Tserpes and Papanikos (2005).

In order to compare the nanotube traction and bending Young moduli, the ratio $E_{S W C N T}^{B} / E_{S W C N T}^{T}$ is studied as a function of the diameter and the nanotube chirality (see Fig. 13). For diameter values greater than $0.5 \mathrm{~nm}$, the difference is less than $2 \%$. Therefore, within an accuracy of $2 \%$, the nanotube Young's modulus can be derived indifferently from its overall traction or bending stiffness.

Thus, we are led to propose the following approximation:

$$
E_{S W C N T}^{B} \approx E_{S W C N T}^{T} \approx E_{\text {graphite }}
$$

which will be more accurate for large-diameter values.

\section{EQUIVALENT SHEAR MODULUS OF SWCNTS}

The shear modulus of SWCNTs is computed from its overall stiffness in torsion. So the polar moment of inertia of the cross section has to be defined, and once again (see the previous section), the expression used for this geometric property is proportional to the graphene thickness $t$. Thus, the shear modulus is defined as

$$
G_{S W C N T}=\frac{K_{T T}^{\mathrm{hom}}}{2 \pi\left(\frac{D_{\text {exact }}}{2}\right)^{3} t} .
$$

The variation of the SWCNT shear modulus as a function of the tube diameter and chirality is illustrated in Fig. 14. The shear modulus value for graphene is also recorded. The shear modulus behaves similarly to the Young's modulus in that it increases with increasing tube diameter. Moreover, for large diameters, the shear modulus becomes insensitive to tube diameter and converges to the graphene sheet value $0.49 \mathrm{TPa}$. However, for small diameters, for example diameters less than $1.5 \mathrm{~nm}$, the shear modulus exhibits a stronger dependence on the diameter than that of the Young's modulus. In contrast to Young's modulus results for a given tube diameter, the shear modulus for zigzag nanotubes is slightly larger than that for armchair nanotubes. Similar variations of the shear modulus with regard to diameter and chirality have been obtained by Tserpes and Papanikos (2005) and Li and Chou (2003) for armchair, zigzag, and chiral SWCNTs.

To summarize, for a variation of the tube diameter between 0.5 and $3.0 \mathrm{~nm}$, the shear modulus of both types of SWCNTs varied from almost 0.445 to $0.495 \mathrm{TPa}$ compared to the graphene sheet value of $0.49 \mathrm{TPa}$. Hence the following approximation resulted, which is a stronger approximation than Eq. (5.6) proposed for the Young's modulus:

$$
G_{S W C N T} \approx G_{\text {graphite }}
$$

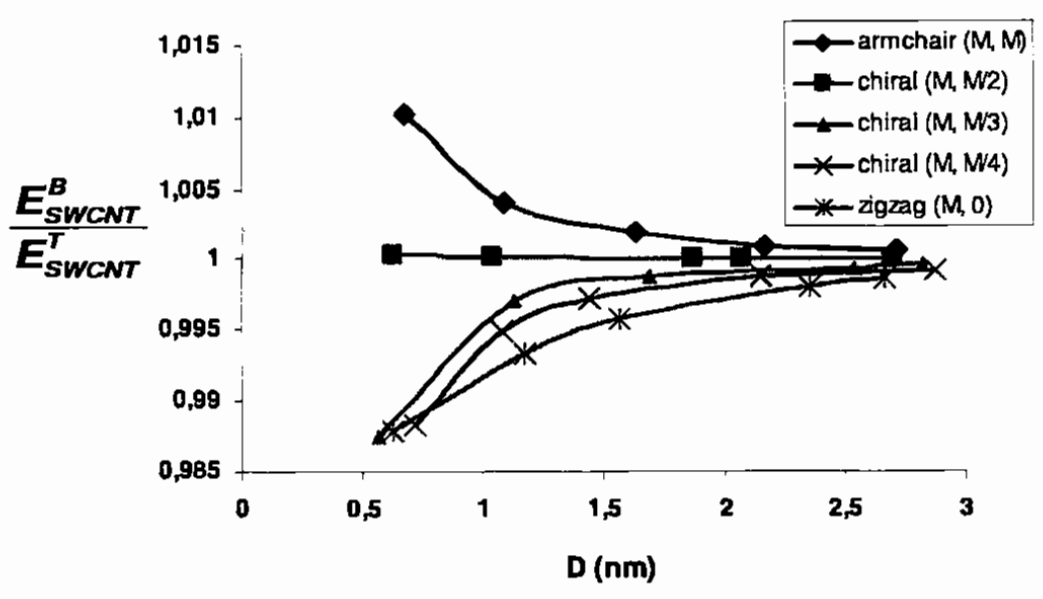

FIG. 13: Variation of Young's modulus from the tensile and bending stiffness 


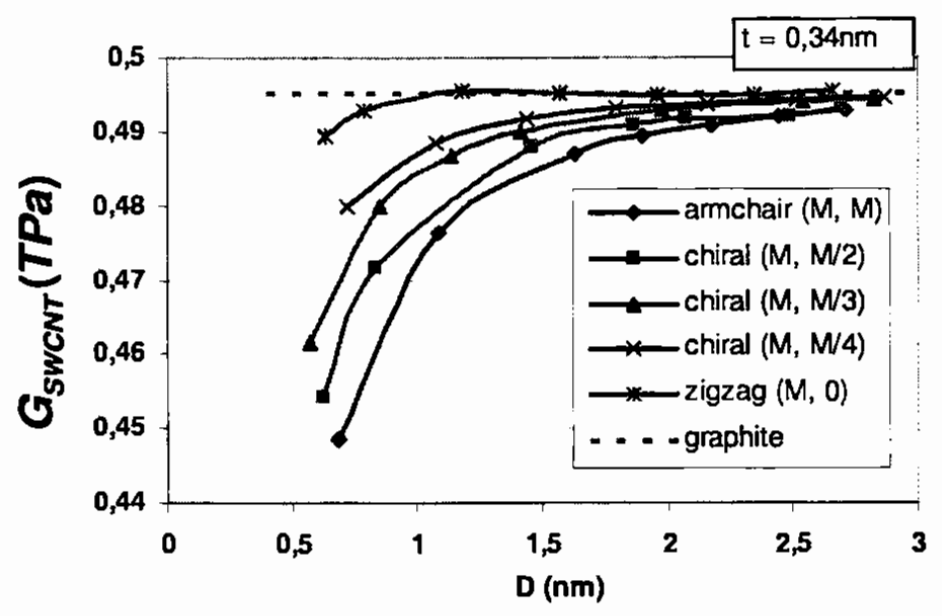

FIG. 14: Variation of shear modulus of different types of SWCNTs with tube diameter compared to the graphite value

\subsection{Comparison with Literature Data}

From the previous results, it can be claimed that the homogenization method has been validated. It has actually been checked that the homogenized stiffnesses agree with those obtained from a macroscopic model (see Table 3) and that equivalent moduli of the SWCNTs tend to be similar to those of the graphene sheet as the diameter increases. However, it is interesting to compare our results to those reported in the literature.

Our computational results and corresponding tendencies are comparable with those obtained from theoretical and experimental studies discussed in Section 3.2 and detailed in Tables 1 and 2. Our results are in very good agreement with those given by theoretical approaches, as can be seen in Hernandez et al. (1998), Li and Chou (2003), Tserpes and Papanikos (2005), Kasti (2007), Chang and Gao (2003), Xiao et al.(2006), and To (2006). The Young's and shear moduli variation was in the range of $1-3 \%$, which can be considered negligible. However, in the works of Meo and Rossi (2006), Giannopoulos et al. (2008), Zhang and Dumitrica (2008), Natsuki et al. (2004), and Agrawal et al.(2006), this variation reaches $8-14 \%$. The reason for this difference could be found in the different potentials used.

Furthermore, the Young's modulus evaluated in the present work is in good agreement with the experimental results (see Table 1) reported in Krishnan et al. (1998), and Salvetat et al. (1999). As mentioned in Section 3.2, difficulty still exists in experimental techniques which measure the torsion response of nanotubes.

Figures 12 and 14 show an increase in Young's and shear moduli with increasing tube diameter. These values converge with those of a graphene sheet for large diameter, as demonstrated in Sections 5.4 and 5.5. Based on structural mechanics, Li and Chou (2003), Chang et al. (2005), Tserpes and Papanikos (2005), Meo and Rossi (2006), To (2006), and Xiao et al. (2006) found similar variation trends of Young's and shear moduli of SWCNTs with those diameters. Moreover, Hernandez et al. (1998) and Zhang and Dumitrica (2008), using TB simulations, also showed an increase in elastic moduli with increase in diameter for small diameter and a quasi-constant evolution for large diameter. We also note that the reverse trend, i.e., a decrease in elastic moduli with diameter, has been noted [see for example, Srivastava et al. (2003), Natsuki et al. (2004), and Agrawal et al. (2006)].

\section{CONCLUSIONS}

This work devoted to the computation of the overall elastic mechanical properties of SWCNTs is based on the homogenization theory. In contrast with most computational approaches reported in the literature where large models of the nanotube are computed, this rigorous mathematical approach enables us to determine the overall behavior of these periodic structures by using microscopic FE models with a reduced number of degrees of freedom. The bending stiffness is thus deducted from an axial basic cell model. Moreover, taking benefit from the two helical symmetries formed by the hexagonal patterns representing the atomic bonds, the mechanical axial behavior can be obtained using a very concise $\mathrm{FE}$ model representing a half hexagon. 
The calculus of SWCNT mechanical properties performed, inducing very reduced computational times, demonstrated that our reduced FE models lead to equivalent elastic moduli comparable to those obtained from other complex modelling techniques. Armchair, zigzag, and chiral SWCNTs were included in the investigation.

The overall beam behavior of the SWCNT was found to be diagonal, except for a traction-torsion coupling for chiral nanotubes. Moreover, this study demonstrated the isotropy of the flexural behavior. From these results, equivalent material moduli were defined, and it was shown that the difference between the traction end bending Young's moduli is very small and tends to zero as the diameter increases.

It appears that for medium and large diameters (more than $1 \mathrm{~nm}$ ), these Young and shear moduli are very close to those of a graphene sheet, both in axial and bending loadings. In that way, the corresponding stiffness can be deduced from the simple analytical Bernouilli-beam formula. By contrast, this work has shown scale-effects for small chiral index nanotubes; the equivalent moduli are sensitive to diameter - about $7 \%$ reduction with respect to graphene. Armchair nanotubes proved to provide slightly higher values of Young's modulus and lower values of shear modulus than zigzag nanotubes for small diameter values.

The developed homogenized approach will be applied soon to the computation of multiwalled carbon nanotubes using basic cell FE models integrating the effects of the interwall nonlinear forces of van der Waals. Moreover, our work will focus next on improvement of the microscopic behavior representation of the carbon atomic interactions and will evaluate the influence of geometric nonlinearity due to large displacements.

\section{REFERENCES}

Agrawal, P. M., Sudalayandi, B. S., Raff, L. M., and Komanduri, R., A comparison of different methods of Young's modulus determination for single-wall carbon nanotubes (SWCNT) using molecular dynamics (MD) simulations, Comput. Mater. Sci., vol. 38, no. 2, pp. 271-281, 2006.

Barros, E., Jorio, A., Samsonidze, G., Capaz, R., Souza Filho, A., Filho, J. M., Dresselhauss, G., and Dresselhauss, M. S., Review on the symmetry-related properties of carbon nanotubes, Phys. Rep., vol. 431, no. 6, pp. 261-302, 2006.

Bendsoe, M. P., Optimal shape design as a material distribution problem, Struct. Multidiscip. Optim., vol. 1, no. 4, pp. 193-202, 1989.

Boutin, C. and Hans, S., Homogenization of periodic discrete medium: Application to dynamics of framed structures, Comput. Geotech., vol. 30, no. 4, pp. 303-320, 2003.

Boutin, C. and Hans, S., Dynamics of discrete framed structures: A unified homogenized description, J. Mech. Mater. Struct., vol. 3, no. 9, pp. 1709-1739, 2008.

Buannic, N. and Cartraud, P., Higher-order effective modelling of periodic heterogeneous beams - Part I: Asymptotic expansion method, Int. J. Solids Struct., vol. 38, no. 40-41, pp. 7139-7161, 2001.

Caillerie, D., Mourad, A., and Raoult, A., Discrete homogenization in graphene sheet modeling, J. Elasticity, vol. 84, no. 1, pp. 33-68, 2006.

Cartraud, P. and Messager, T., Computational homogenization of periodic beam-like structures, Int. J. Solids Struct., vol. 43, no. 3-4, pp. 686-696, 2006.

Chang, T. and Gao, H., Size-dependent elastic properties of a single-walled carbon nanotube via a molecular mechanics model, $J$. Mech. Phys. Solids, vol. 51, no. 6, pp. 1059-1074, 2003.

Chang, T., Geng, J., and Guo, X., Chirality- and size-dependent elastic properties of single-walled carbon nanotubes, Appl. Phys. Lett., vol. 87, no. 25, p. 251929, 2005.

Cornell, W. D., Cieplak, P., Bayly, C. I., Gould, I. R., Merz, K. M., and Ferguson, D. M., A second generation force-field for the simulation of proteins, nucleic acids and organic molecules, J. Am. Chem. Soc., vol. 117, pp. 5179-5197, 1995.

Dallot, J., Sab, K., and Foret, G., Limit analysis of periodic beams, Eur. J. Mech., vol. 28, no. 1, pp. 166-178, 2009.

Dresselhaus, M. S., Dresselhaus, G., and Avouris, P., Carbon nanotubes (synthesis, structure properties and applications), Top. Appl. Phys., vol. 80, pp. 329-390, 2001.

Dumitrica, T. and James, R. D., Objective molecular dynamics, J. Mech. Phys. Solids, vol. 55, no. 10, pp. 2206-2236, 2007.

Geers, M. G. D., Coenen, E. W. C., and Kouznetsova, V. G., Multi-scale computational homogenization of structured thin sheets, Modelling Simul. Mater. Sci. Eng., vol. 15, no. 4, pp. 393-404, 2007. 
Giannopoulos, G. I., Kakavas, P. A., and Anifantis, N. K., Evaluation of the effective mechanical properties of single walled carbon nanotubes using a spring based finite element approach, Comput. Mater. Sci., vol. 41, no. 4, pp. 561-569, 2008.

Gupta, S., Dharamvir, K., and Jindal, V. K., Elastic moduli of single-walled carbon nanotubes and their ropes, Phys. Rev. B, vol. 72, no. 16, 2005.

Gupta, S. S. and Batra, R. C., Continuum structures equivalent in normal mode vibrations to single-walled carbon nanotubes, Comput. Mater. Sci., vol. 43, pp. 715-723, 2008.

Hernandez, E., Goze, C., Bernier, P., and Rubio, A., Elastic properties of $\mathrm{C}$ and $\mathrm{B}_{x} \mathrm{C}_{y} \mathrm{~N}_{z}$ composite nanotubes, Phys. Rev. Lett., vol. 80, no. 20, pp. 4502-4505, 1998.

Huang, Y., Wu, J., and Hwang, K. C., Thickness of graphene and single-wall carbon nanotubes, Phys. Rev. B, vol. 74, no. $24,2006$.

Kalamkarov, A. L., Georgiades, A. V., Rokkam, S. K., Veedu, V. P., and Ghasemi-Nejhad, M. N., Analytical and numerical techniques to predict carbon nanotubes properties, Int. J. Solids Struct., vol. 43, no. 22-23, pp. 6823-6854, 2006.

Kasti, N., Zigzag carbon nanotubes-Molecular/structural mechanics and the finite element method, Int. J. Solids Struct., vol. 44, no. 21, pp. 6914-6929, 2007.

Krishnan, A., Dujardin, E., Ebbessen, T. W., and Yianilos et Treacy, M., Young's modulus of single-walled nanotubes, Phys. Rev. $B$, vol. 58, no. 20, 1998.

Léné, F., Contribution à l'étude des Matériaux Composites et de Leur Endommagement, Thèse d'Etat, Universite Pierre et Marie Curie, Paris, 1984.

$\mathrm{Li}, \mathrm{C}$. and Chou, T. W., A structural mechanics approach for the analysis of carbon nanotubes, Int. J. Solids Struct., vol. 40, no. 10, pp. 2487-2499, 2003.

Liu, W. K., Karpov, E. G., and Park, H. S., Nano Mechanics and Materials; Theory Multiscale Methods and Applications, John Wiley \& Sons, England, 2006.

Lu, J. P., Elastic properties of carbon nanotubes and nanoropes, Phys. Rev. Lett., vol. 79, no. 7, pp. 1297-1300, 1997.

Meo, M. and Rossi, M., Prediction of Young's modulus of single wall carbon nanotubes by molecular-mechanics based finite element modelling, Compos. Sci. Technol., vol. 66, no. 11-12, pp. 1597-1605, 2006.

Messager, T. and Cartraud, P., Homogenization of helical beam-like structures: Application to single-walled carbon nanotubes, Comput. Mech., vol. 41, no. 2, pp. 335-346, 2008.

Mintmire, J. W. and White, C. T., Electronic and structural properties of carbon nanotubes, Carbon, vol. 33, no. 7, pp. 893-902, 1995.

Moustaghfir, N., Daya, E. M., Braikat, B., Damil, N., and Potier-Ferry, M., Evaluation of continuous modelings for the modulated vibration modes of long repetitive structures, Int. J. Solids Struct., vol. 44, no. 21, pp. 7061-7072, 2007.

Natsuki, T., Tantrakarn, K., and Endo, M., Effect of carbon nanotube structures on mechanical properties, Appl. Phys. A, vol. 79, no. 1, pp. 117-124, 2004.

Odegard, G. M., Gates, T. S., Nicholson, L. M., and Wise, K. E., Equivalent-continuum modeling of nano-structured materials, Compos. Sci. Technol., vol. 62, no. 14, pp. 1869-1880, 2002.

Pantano, A., Parks, D. M., and Boyce, M. C., Mechanics of deformation of single- and multi-wall carbon nanotubes, J. Mech. Phys. Solids, vol. 52, pp. 789-821, 2004.

Qian, D., Wagner, G. J., Liu, W. K., Yu, M. F., and Ruoff, R. S., Mechanics of carbon nanotubes, Appl. Mech. Rev., vol. 55, no. 6, pp. 495-533, 2002.

Rafii-Tabar, H., Computational modelling of thermo-mechanical and transport properties of carbon nanotubes, Phys. Rep., vol. 390, pp. 235-452, 2004.

Rappe, A. K., Casemit, C. J., Colwell, K. S., Goddard, W. A., and Skiff, W. M., UFF, A full periodic-table force field for molecular mechanics and molecular dynamics simulations, J. Am. Chem. Soc., vol. 114, pp. 24-35, 1992.

Reddy, C. D., Rajendran, S., and Liew, K. M., Equilibrium configuration and continuum elastic properties of finite sized graphene, Nanotechnology, vol. 17, pp. 864-870, 2006.

Salvetat, J. P., Briggs, A. D., Bonard, J. M., Basca, R. R., Kulik, A. J., Stockli, T., Burnham, N. A., and Forro, L., Elastic and shear moduli of single-walled carbon nanotube ropes, Phys. Rev. Lett., vol. 82, pp. 944-947, 1999.

Srivastava, D., Wei, C., and Cho, K., Computational nanomechanics of carbon nanotubes and composites, Appl. Mech. Rev., vol. 56, no. 2, pp. 215-230, 2003. 
To, W. S., Bending and shear moduli of single-walled carbon nanotubes, Finite Elements Analysis Design, vol. 42, pp. 404-413, 2006.

Trabucho, L. and Viaño, J. M., Mathematical Modelling of Rods, Eds. Ciarlet, P. G. and Lions, J. L., Handbook of Numerical Analysis, North-Holland, Amsterdam, vol. 4, pp. 487-973, 1996.

Tserpes, K. I. and Papanikos, P., Finite element modelling of single-walled carbon nanotubes, Composites, Part B, vol. 36, pp. 468-477, 2005.

Tu, Z. C. and Ou-Yang, Z. C., Elastic theory of low-dimensional continua and its applications in bio- and nano-structures, $J$. Comput. Theor: Nanosci., vol. 5, pp. 422-448, 2008.

Van Lier, G., Van Alsenoy, C., Van Doren, V., and Geerlings, P., Ab initio study of the elastic properties of single-walled carbon nanotubes and grapheme, Chem. Phys. Lett., vol. 326, pp. 181-185, 2000.

Wang, Q. and Varadan, V. K., Wave characteristics of carbon nanotubes, Int. J. Solids Struct, vol. 43, no. 2, pp. 254-265, 2005.

Wenxing, B., Changchun, Z., and Wanzhao, C., Simulation of Young's modulus of single-walled carbon nanotubes by molecular dynamics, Physica B, vol. 352, pp. 156-163, 2004.

Wu, Y., Zhang, X., Leung, A. Y. T., and Zhong, W., An energy-equivalent model on studying the mechanical properties of singlewalled carbon nanotubes, Thin-Walled Structures, vol. 44, no. 6, pp. 667-676, 2006.

Xiao, J. R., Lopatnikov, S. L., Gama, B. A., and Gillespie, J. W., Nanomechanics of the deformation of single- and multi-walled carbon nanotubes under radial pressure, Mater. Sci. Eng., A, vol. 416, pp. 192-204, 2006.

Zhang, D.-B. and Dumitrica, T., Elasticity of ideal single-walled carbon nanotubes via symmetry-adapted tight-binding objective modeling, Appl. Phys. Lett., vol. 93, 2008.

\section{APPENDIX}

In the plane of the graphene sheet, the nanotube axis is orthogonal to $\overrightarrow{O O}^{\prime}$. Therefore, the nanotube axis aligns to the translational vector

$$
\vec{T}=t_{1} \vec{a}_{M}+t_{2} \vec{a}_{N}
$$

where $t_{1}$ and $t_{2}$ are related to the chiral indices:

$$
\left\{\begin{array}{l}
t_{1}=(M+2 N) / d_{R} \\
t_{2}=-(2 M+N) / d_{R}
\end{array}\right.
$$

$d_{R}$ being the highest common divisor of $(M+2 N, 2 M+N)$. For the SWCNT $(M=4, N=2)$ example shown in Fig. 1 , we have $d_{R}=2$, and the axial periodicity translation was found to be $\vec{T}=4 \vec{a}_{M}-5 \vec{a}_{N}$. For armchair and zigzag chiral tubes, we obtain general forms for this translation vector representing the axial periodicity: for armchair $(M, M), \vec{T}=\vec{a}_{M}-\vec{a}_{N}$, while for zigzag SWCNTs $(M, 0)$ it is $\vec{a}_{M}-2 \vec{a}_{N}$. The axial cell of SWCNT is delineated by the vectors $\vec{T}$ and $\overrightarrow{O O^{\prime}}$ (see Fig. 1). The area of the nanotube axial cell can be easily calculated as a vector-product of these two vectors (Mintmire and White, 1995):

$$
\left\|\overrightarrow{O O^{\prime}} \wedge \vec{T}\right\|=\sqrt{3}\left(\alpha_{C-C}\right)^{2}\left(M^{2}+M \cdot N+N^{2}\right) / d_{R}
$$

By dividing this product by the area of the axial cell of a graphene layer,

$$
\left\|\vec{a}_{M} \wedge \vec{a}_{N}\right\|=\sqrt{3}\left(\alpha_{C-C}\right)^{2} / 2
$$

one can get the number of hexagons in the axial cell of a nanotube (Barros et al., 2006):

$$
N b=\frac{2\left(M^{2}+M N+N^{2}\right)}{d_{R}} .
$$

The length of the translational vector is given by

$$
T=\frac{3 \alpha_{C-C}}{d_{R}} \sqrt{M^{2}+N^{2}+M N} .
$$

An example of an axial period of chiral SWCNT is presented in Fig. 10. 